\title{
Lepton pair production in ultraperipheral collisions
}

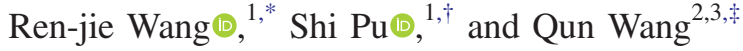 \\ ${ }^{1}$ Department of Modern Physics, University of Science and Technology of China, Anhui 230026, China \\ ${ }^{2}$ Interdisciplinary Center for Theoretical Study and Department of Modern Physics, University of Science \\ and Technology of China, Hefei, Anhui 230026, China \\ ${ }^{3}$ Peng Huanwu Center for Fundamental Theory, Hefei, Anhui 230026, China
}

(Received 16 June 2021; accepted 11 August 2021; published 13 September 2021)

\begin{abstract}
The lepton pair production in ultraperipheral collisions is studied in the classical field approximation. We derive a general form of the cross section in terms of photon distributions that depend on the transverse momentum and coordinate based on the wave packet form of nuclear wave functions. Such a general form of the cross section in the classical field approximation contains the results of the generalized equivalent photon approximation (EPA) as well as the corrections beyond EPA in the Born approximation. By rewriting the general form of the cross section in light-cone coordinates, we find a good connection with the transverse momentum dependent distribution factorization formalism in the Born approximation. Our numerical results are consistent with current experimental data.
\end{abstract}

DOI: 10.1103/PhysRevD.104.056011

\section{INTRODUCTION}

Strong electromagnetic fields are produced in the relativistic heavy ion collisions [1-9]. One way to describe the evolution of electromagnetic fields is through the relativistic magnetohydrodynamics consisting of the hydrodynamical conservation equations coupled with Maxwell's equations. The solutions to equations of ideal magnetohydrodynamics with longitudinal boost invariance show that the transverse magnetic field decays as $\sim 1 / \tau$ with $\tau$ being the proper time [10-15] (for a recent numerical simulation of electromagnetic fields based on ideal magnetohydrodynamics, see, e.g., Ref. [8]).

Although strong electromagnetic fields decay rapidly, there are still many novel transport phenomena induced by strong fields that could be measured in experiments, such as the chiral magnetic and separation effects $[1,16,17]$, the chiral electric separation effect [18-21], and other nonlinear chiral transport phenomena [21-25]. These effects can be described by chiral kinetic theory for massless fermions derived from the path integral [26-28], the Hamiltonian approaches [29,30], the quantum kinetic theory via Wigner functions [23,31-38], and the worldline formalism [39]. The chiral kinetic theory has been

\footnotetext{
*wrjn@mail.ustc.edu.cn

†shipu@ustc.edu.cn

qunwang@ustc.edu.cn
}

Published by the American Physical Society under the terms of the Creative Commons Attribution 4.0 International license. Further distribution of this work must maintain attribution to the author(s) and the published article's title, journal citation, and DOI. Funded by SCOAP ${ }^{3}$. extended to the massive fermions and with collision kernels [40-48]. For reviews of recent developments in this field, see, e.g., Refs. [49-53].

Another type of effects is related to the nonperturbative production of lepton pairs in strong electric fields through the Schwinger mechanism [54]. Recent developments along this line include the lepton pair production in strong magnetic fields by the Schwinger mechanism [55-58] and the vacuum birefringence [59-61].

Recently the lepton pair production through strong electromagnetic fields in ultraperipheral collisions (UPC) has drawn broad interest. Back to 1930s, Weizsacker and Williams considered the electromagnetic field produced by a fast moving particle as an equivalent flux of quasireal photons $[62,63]$. This approximation is called the Weizsacker-Williams method or equivalent photon approximation (EPA) [64]. A related process is the lepton pair production through collisions of two real photons and was studied by Breit and Wheeler [65] under the condition that the total energy of two photons should be greater than the mass of the lepton pair. The STAR Collaboration at Relativistic Heavy Ion Collider (RHIC) has measured the lepton pair $(l \bar{l})$ production process in UPC [66]. There are also several measurements related to nonlinear effects of quantum electrodynamics (QED) such as the vacuum birefringence [67] and the light-bylight scattering [68]. The transverse momentum spectra of the lepton pair in peripheral collisions of heavy ions are found to be significantly broader than the ones in UPC by STAR [69] and by the ATLAS Collaboration at the Large Hadron Collider (LHC) [70]. Such broadenings may arise from medium effects in peripheral collisions; therefore, 
UPC may provide a baseline for future studies of medium effects.

Several theoretical methods are available to describe the lepton pair production in UPC. A widely used method is the EPA or the generalized EPA (gEPA). The total cross section of $\gamma \gamma \rightarrow l \bar{l}$ has been calculated in EPA in the classical field approximation of QED [71]. The original EPA calculations predict that the position (transverse momentum) of the peak in the transverse momentum spectrum is less than $20 \mathrm{MeV}$, inconsistent with the experimental data [72-74]. Therefore the generalized EPA was proposed [71,74-79] to give the correct position of the peak in the transverse momentum spectrum [77-79].

The azimuthal asymmetry in the lepton pair from linearly polarized photons in UPC has been studied in the transverse momentum dependent (TMD) factorization formalism [80,81] similar to polarized gluons [82-84]. In this formalism, the photon Wigner functions are introduced into the cross section which depends on the transverse momentum and coordinate $[85,86]$. The broadening of transverse momentum has also been studied in the TMD formalism [87].

With all these different formulations in different perspectives of the process, it is natural to ask if there is a unified description. In this paper, we will derive a general form of the cross section based on wave-packet nuclear wave functions, which incorporates photon distributions with the dependence on the transverse momentum and coordinate. The cross sections in (g)EPA and the TMD formalism can be derived from the general form. The numerical results of the cross section in the general form are in good agreement with experimental data.

This paper is organized as follows. In Sec. II, we derive a general form of the cross section in terms of transverse momentum and coordinate dependent photon distributions. In Sec. III, we implement the classical field approximation. In Sec. IV we take the ultrarelativistic limit to reproduce the results of (g)EPA. In Sec. V, we rewrite the general form of the cross section in light-cone coordinates and make a connection with results of the TMD formalism. The numerical results of the cross section in the general form are given in Sec. VI, and a comparison is made with experimental data as well as the results of (g)EPA and the
TMD formalism. The main results of this work are summarized in Sec. VII.

Throughout this paper, we choose the metric $g_{\mu \nu}=$ $\operatorname{diag}\{+,-,-,-\}$ for ordinary coordinates $\left(x^{0}, x^{1}, x^{2}, x^{3}\right)=$ $(t, \mathbf{x})$. The light-cone coordinates $x^{\mu}=\left(x^{+}, x^{-}, \mathbf{x}_{T}\right)$ with $x^{ \pm}=\left(x^{0} \pm x^{3}\right) / \sqrt{2}$ and $\mathbf{x}_{T}=\left(x^{1}, x^{2}\right)$ are also used. A vector $a^{\mu}$ can be written as $a^{\mu}=a^{+} n_{+}^{\mu}+a^{-} n_{-}^{\mu}+a_{T}^{\mu}$ where $a^{ \pm}=\left(a^{0} \pm a^{3}\right) / \sqrt{2}$ and $n_{ \pm}^{\mu}$ are lightlike vectors satisfying $n_{+}^{2}=n_{-}^{2}=0$ and $n_{+} \cdot n_{-}=1$. The inner product of two vectors in light-cone coordinates is $a \cdot b=a^{-} b^{+}+a^{+} b^{-}-a_{T} \cdot b_{T}$.

\section{GENERAL FORM OF CROSS SECTIONS FOR LEPTON PAIRS}

In this section, we give a general form for the differential cross section of lepton pairs in UPC, with the detailed derivation being given in the Appendix A.

As shown in Fig. 2, we consider collisions of two nuclei $A_{1}$ and $A_{2}$ moving alone in the $\pm z$ direction which are displaced by an impact parameter $\mathbf{b}_{T}$ and generate a pair of leptons $l$ and $\bar{l}$ along with other particles $X_{1}, \ldots, X_{f}, \ldots$,

$A_{1}\left(P_{A 1}\right)+A_{2}\left(P_{A 2}\right) \rightarrow l\left(k_{1}\right)+\bar{l}\left(k_{2}\right)+\sum_{f} X_{f}\left(K_{f}\right)$,

where four-momenta of particles are given in parentheses. Here $P_{A 1}^{\mu}=\left(E_{A 1}, \mathbf{P}_{A 1}\right)$ and $P_{A 2}^{\mu}=\left(E_{A 2}, \mathbf{P}_{A 2}\right)$ with $E_{A 1}=$ $\sqrt{\mathbf{P}_{A 1}^{2}+M_{1}^{2}}$ and $E_{A 2}=\sqrt{\mathbf{P}_{A 2}^{2}+M_{2}^{2}}$ are on-shell momenta of two nuclei with masses $M_{1}$ and $M_{2}$, respectively. The three-momenta of two nuclei are $\mathbf{P}_{A 1}=\left(0,0, P_{A 1}^{z}\right)$ and $\mathbf{P}_{A 2}=\left(0,0,-P_{A 1}^{z}\right)$ in the center of mass frame of the collision.

In order to describe collisions at fixed impact parameters, we need to assume the wave functions of colliding nuclei to be wave packets. We follow the standard way in quantum field theory to obtain the cross section at the impact parameter $\mathbf{b}_{T}$ in Eq. (A5). Making the ansatz (A9) for the longitudinal momentum amplitudes of wave packets and completing the integrals over longitudinal momenta of wave packets we arrive at

$$
\begin{aligned}
\sigma= & \frac{1}{8(2 \pi)^{8}} \int d^{2} \mathbf{b}_{T} d^{2} \mathbf{b}_{1 T} d^{2} \mathbf{b}_{2 T} \sum_{\{f\}} \int \frac{d^{3} k_{1}}{(2 \pi)^{3} 2 E_{k 1}} \frac{d^{3} k_{2}}{(2 \pi)^{3} 2 E_{k 2}} \prod_{f} \frac{d^{3} K_{f}}{(2 \pi)^{3} 2 E_{K f}} \\
& \times \int d^{2} \mathbf{P}_{1 T} d^{2} \mathbf{P}_{2 T} d^{2} \mathbf{P}_{1 T}^{\prime} d^{2} \mathbf{P}_{2 T}^{\prime} \frac{1}{v \sqrt{E_{P 1} E_{P 2} E_{P 1^{\prime}} E_{P 2^{\prime}}}} G^{2}\left[\left(P_{1}^{z}-P_{A 1}^{z}\right)^{2}\right] \phi_{T}\left(\mathbf{P}_{1 T}\right) \phi_{T}\left(\mathbf{P}_{2 T}\right) \phi_{T}^{*}\left(\mathbf{P}_{1 T}^{\prime}\right) \phi_{T}^{*}\left(\mathbf{P}_{2 T}^{\prime}\right) \\
& \times e^{-i \mathbf{b}_{1 T} \cdot \mathbf{\Delta}_{1 T}} e^{-i \mathbf{b}_{2 T} \cdot \mathbf{\Delta}_{2 T}} \delta^{(2)}\left(\mathbf{b}_{T}-\mathbf{b}_{1 T}+\mathbf{b}_{2 T}\right)(2 \pi)^{4} \delta^{(4)}\left(P_{1}+P_{2}-k_{1}-k_{2}-\sum_{f} K_{f}\right) \\
& \times \sum_{\operatorname{spin} \text { of } l, \bar{l}} \mathcal{M}_{P_{1}+P_{2} \rightarrow k_{1}+k_{2}+\sum_{f} K_{f}} \mathcal{M}_{P_{1}^{\prime}+P_{2}^{\prime} \rightarrow k_{1}+k_{2}+\sum_{f} K_{f}}^{*}
\end{aligned}
$$



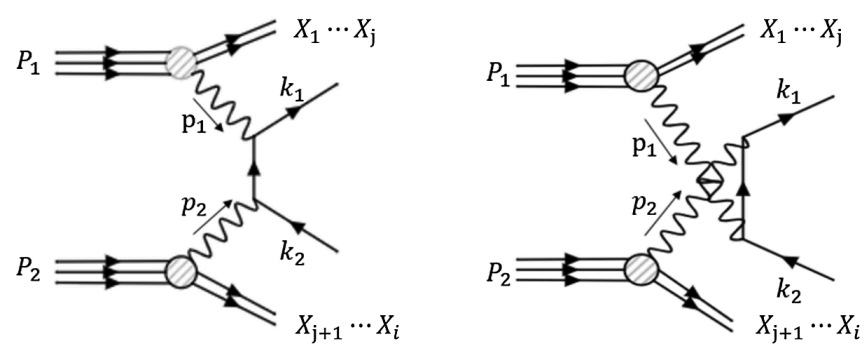

FIG. 1. Feynman diagrams for the photon fusion process in UPC.

Here $P_{1}, P_{2}, P_{1}^{\prime}$, and $P_{2}^{\prime}$ are on-shell momenta of nuclear wave packets given by $P_{1}=\left(E_{P 1}, \mathbf{P}_{1 T}, 2 P_{A 1}^{z}-P_{1}^{\prime z}\right)$, $P_{2}=\left(E_{P 2}, \mathbf{P}_{2 T},-2 P_{A 1}^{z}+P_{1}^{\prime z}\right), P_{1}^{\prime}=\left(E_{P 1^{\prime}}, \mathbf{P}_{1 T}^{\prime}, P_{1}^{\prime z}\right)$, and $P_{2}^{\prime}=\left(E_{P 2^{\prime}}, \mathbf{P}_{2 T}^{\prime},-P_{1}^{\prime z}\right)$. As the solution to the energy conservation $P_{1}^{\prime z}$ is a function of transverse momenta $\mathbf{P}_{1 T}, \mathbf{P}_{2 T}, \mathbf{P}_{1 T}^{\prime}$, and $\mathbf{P}_{2 T}^{\prime}$. We see that the $z$ components of $P_{1}+P_{2}$ and $P_{1}^{\prime}+P_{2}^{\prime}$ are vanishing. The function $G\left(x^{2}\right)$ is defined in Eq. (A9), which is a positive and decreasing function of $x^{2}$ satisfying $G(0)=1$. We have used the shifts of transverse momenta $\boldsymbol{\Delta}_{1 T} \equiv \mathbf{P}_{1 T}^{\prime}-\mathbf{P}_{1 T}$ and $\boldsymbol{\Delta}_{2 T} \equiv \mathbf{P}_{2 T}^{\prime}-\mathbf{P}_{2 T}$, and the relative velocity of two nuclei $v$ given in Eq. (A10) as a function of transverse momenta.

In the tree level of Feynman diagrams (sometimes called Born approximation) as shown in Fig. 1, the lepton pair is produced in the photon fusion process

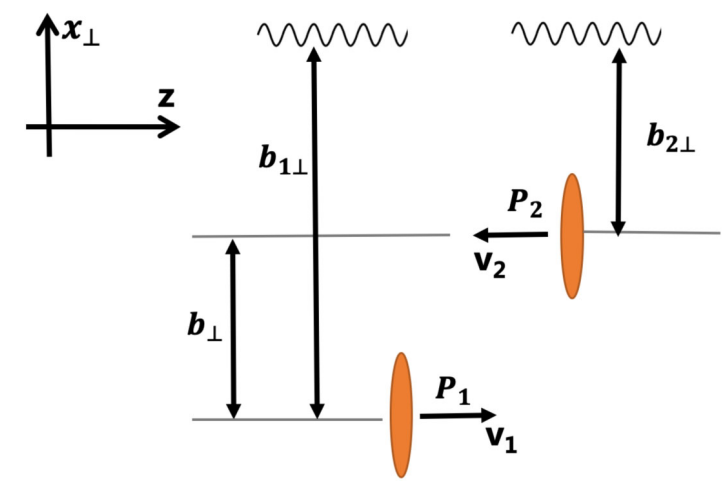

FIG. 2. A cartoon for photon emission in UPC.

$$
\gamma\left(p_{1}\right)+\gamma\left(p_{2}\right) \rightarrow l\left(k_{1}\right)+\bar{l}\left(k_{2}\right)
$$

Here, we assume the photon $\gamma\left(p_{1}\right)$ or $\gamma\left(p_{2}\right)$ comes from the nuclei $A_{1}\left(P_{1}\right)$ or $A_{2}\left(P_{2}\right)$, respectively. Note that each photon does not have to come from the nuclear center. We can identify $\mathbf{b}_{i T}$ in Eq. (2) as the transverse distance between $\gamma\left(p_{i}\right)$ and $A_{i}\left(P_{i}\right)$ with $i=1,2$, which are related to the impact parameter $\mathbf{b}_{T}=\mathbf{b}_{1 T}-\mathbf{b}_{2 T}$ as shown in Fig. 2.

The invariant amplitude $\mathcal{M}$ can be obtained through the matrix element of the operator $\hat{T}$ or the $T$-matrix element. The results are given in Eqs. (A16) and (A17). By inserting these results for invariant amplitudes into Eq. (2), the differential cross section can be put into the form

$$
\begin{aligned}
\frac{d \sigma}{d^{3} k_{1} d^{3} k_{2}} \approx & \frac{1}{32(2 \pi)^{6}} \frac{1}{E_{k 1} E_{k 2}} \int d^{2} \mathbf{b}_{T} d^{2} \mathbf{b}_{1 T} d^{2} \mathbf{b}_{2 T} \int d^{4} p_{1} d^{4} p_{2} \delta^{(2)}\left(\mathbf{b}_{T}-\mathbf{b}_{1 T}+\mathbf{b}_{2 T}\right)(2 \pi)^{4} \delta^{(4)}\left(p_{1}+p_{2}-k_{1}-k_{2}\right) \\
& \times \int \frac{d^{2} \mathbf{P}_{\left(1+1^{\prime}\right) T}}{(2 \pi)^{2}} \frac{d^{2} \mathbf{P}_{\left(2+2^{\prime}\right) T}}{(2 \pi)^{2}} \frac{1}{v \sqrt{E_{P 1} E_{P 2} E_{P 1^{\prime}} E_{P 2^{\prime}}}} G^{2}\left[\left(P_{1}^{\prime z}-P_{A 1}^{z}\right)^{2}\right] \phi_{T}\left(\mathbf{P}_{1 T}\right) \phi_{T}\left(\mathbf{P}_{2 T}\right) \phi_{T}^{*}\left(\mathbf{P}_{1 T}^{\prime}\right) \phi_{T}^{*}\left(\mathbf{P}_{2 T}^{\prime}\right) \\
& \times \mathcal{S}_{\sigma \mu}\left(p_{1}, \mathbf{b}_{1 T}\right) \mathcal{S}_{\rho \nu}\left(p_{2}, \mathbf{b}_{2 T}\right) L^{\mu \nu ; \sigma \rho}\left(p_{1}, p_{2} ; p_{1}-P_{1}+P_{1}^{\prime}, p_{2}-P_{2}+P_{2}^{\prime} ; k_{1}, k_{2}\right),
\end{aligned}
$$

where $P_{1}^{\prime z}$ is a function of nuclear transverse momenta that approaches $P_{A 1}^{z}$ if all nuclear transverse momenta are vanishing, $L^{\mu \nu ; \sigma \rho}$ is the lepton tensor in Eq. (A21), and we have used variables $\boldsymbol{\Delta}_{1 T}$ and $\boldsymbol{\Delta}_{2 T}$ with

$$
\begin{aligned}
\mathbf{P}_{\left(1+1^{\prime}\right) T} & =\frac{1}{2}\left(\mathbf{P}_{1 T}+\mathbf{P}_{1 T}^{\prime}\right), \\
\mathbf{P}_{\left(2+2^{\prime}\right) T} & =\frac{1}{2}\left(\mathbf{P}_{2 T}+\mathbf{P}_{2 T}^{\prime}\right),
\end{aligned}
$$

to replace $\mathbf{P}_{1 T}, \mathbf{P}_{2 T}, \mathbf{P}_{1 T}^{\prime}$, and $\mathbf{P}_{2 T}^{\prime}$. We have used the Wigner functions for photons

$$
\begin{aligned}
& \mathcal{S}_{\sigma \mu}\left(p_{1}, \mathbf{b}_{1 T}\right) \equiv \int \frac{d^{2} \boldsymbol{\Delta}_{1 T}}{(2 \pi)^{2}} \int \frac{d^{4} y_{1}}{(2 \pi)^{4}} e^{i p_{1} \cdot y_{1}}\left\langle P_{1}^{\prime}\left|A_{\sigma}^{\dagger}(0) A_{\mu}\left(y_{1}\right)\right| P_{1}\right\rangle e^{-i \mathbf{b}_{1 T} \cdot \mathbf{\Delta}_{1 T}}, \\
& \mathcal{S}_{\rho \nu}\left(p_{2}, \mathbf{b}_{2 T}\right) \equiv \int \frac{d^{2} \boldsymbol{\Delta}_{2 T}}{(2 \pi)^{2}} \int \frac{d^{4} y_{2}}{(2 \pi)^{4}} e^{i p_{2} \cdot y_{2}}\left\langle P_{2}^{\prime}\left|A_{\rho}^{\dagger}(0) A_{\nu}\left(y_{2}\right)\right| P_{2}\right\rangle e^{-i \mathbf{b}_{2 T} \cdot \mathbf{\Delta}_{2 T}},
\end{aligned}
$$

which are similar to those in Refs. $[85,86]$. We will discuss them carefully in the TMD factorization formalism in Sec. V. 
We can also integrate Eq. (4) over $\mathbf{b}_{1 T}$ and $\mathbf{b}_{2 T}$ and express the differential cross section at fixed $\mathbf{b}_{T}$

$$
\begin{aligned}
& \frac{d \sigma}{d^{3} k_{1} d^{3} k_{2} d^{2} \mathbf{b}_{T}}=\frac{1}{32(2 \pi)^{6}} \frac{1}{E_{k 1} E_{k 2}} \int d^{4} p_{1} d^{4} p_{2} \int \frac{d^{2} \mathbf{P}_{1 T}}{(2 \pi)^{2}} \frac{d^{2} \mathbf{P}_{2 T}}{(2 \pi)^{2}} \frac{d^{2} \mathbf{P}_{1 T}^{\prime}}{(2 \pi)^{2}} \frac{d^{2} \mathbf{P}_{2 T}^{\prime}}{(2 \pi)^{2}} \frac{1}{v \sqrt{E_{P 1} E_{P 2} E_{P 1^{\prime}} E_{P 2^{\prime}}}} \\
& \times e^{-i \mathbf{b}_{T} \cdot \boldsymbol{\Delta}_{1 T}} G^{2}\left[\left(P_{1}^{\prime z}-P_{A 1}^{z}\right)^{2}\right] \phi_{T}\left(\mathbf{P}_{1 T}\right) \phi_{T}\left(\mathbf{P}_{2 T}\right) \phi_{T}^{*}\left(\mathbf{P}_{1 T}^{\prime}\right) \phi_{T}^{*}\left(\mathbf{P}_{2 T}^{\prime}\right) \\
& \times L^{\mu \nu ; \sigma \rho}\left(p_{1}, p_{2} ; p_{1}-P_{1}+P_{1}^{\prime}, p_{2}-P_{2}+P_{2}^{\prime} ; k_{1}, k_{2}\right) \int \frac{d^{4} y_{1}}{(2 \pi)^{4}} e^{i p_{1} \cdot y_{1}}\left\langle P_{1}^{\prime}\left|A_{\sigma}^{\dagger}(0) A_{\mu}\left(y_{1}\right)\right| P_{1}\right\rangle \\
& \times \int \frac{d^{4} y_{2}}{(2 \pi)^{4}} e^{i p_{2} \cdot y_{2}}\left\langle P_{2}^{\prime}\left|A_{\rho}^{\dagger}(0) A_{\nu}\left(y_{2}\right)\right| P_{2}\right\rangle(2 \pi)^{2} \delta^{(2)}\left(\mathbf{P}_{1 T}+\mathbf{P}_{2 T}-\mathbf{P}_{1 T}^{\prime}-\mathbf{P}_{2 T}^{\prime}\right)(2 \pi)^{4} \delta^{(4)}\left(p_{1}+p_{2}-k_{1}-k_{2}\right) .
\end{aligned}
$$

We can also integrate Eq. (7) over $\mathbf{b}_{T}$ to obtain

$$
\begin{aligned}
\frac{d \sigma}{d^{3} k_{1} d^{3} k_{2}}= & \frac{1}{32(2 \pi)^{6}} \frac{1}{E_{k 1} E_{k 2}} \int d^{4} p_{1} d^{4} p_{2} \int \frac{d^{2} \mathbf{P}_{1 T}}{(2 \pi)^{2}} \frac{d^{2} \mathbf{P}_{2 T}}{(2 \pi)^{2}} \frac{1}{v E_{P 1} E_{P 2}}\left|\phi_{T}\left(\mathbf{P}_{1 T}\right)\right|^{2}\left|\phi_{T}\left(\mathbf{P}_{2 T}\right)\right|^{2} \\
& \times S_{\sigma \mu}\left(P_{1}, p_{1}\right) S_{\rho \nu}\left(P_{2}, p_{2}\right) L^{\mu \nu ; \sigma \rho}\left(p_{1}, p_{2} ; p_{1}, p_{2} ; k_{1}, k_{2}\right)(2 \pi)^{4} \delta^{4}\left(p_{1}+p_{2}-k_{1}-k_{2}\right)
\end{aligned}
$$

where we have used $P_{1}^{\prime z}=P_{A 1}^{z}$ as the solution to the energy conservation equation with the conditions $\mathbf{P}_{1 T}=\mathbf{P}_{1 T}^{\prime}$ and $\mathbf{P}_{2 T}=\mathbf{P}_{2 T}^{\prime}$, so the on-shell momenta of nuclear wave packets now become $P_{1}=\left(E_{P 1}, \mathbf{P}_{1 T}, P_{A 1}^{z}\right)$ and $P_{2}=\left(E_{P 2}, \mathbf{P}_{2 T},-P_{A 1}^{z}\right)$. We have also defined

$$
S_{\sigma \mu}(P, p) \equiv \int \frac{d^{4} y}{(2 \pi)^{4}} e^{i p \cdot y}\left\langle P\left|A_{\sigma}^{\dagger}(0) A_{\mu}(y)\right| P\right\rangle,
$$

as the TMD correlation function for unpolarized nuclei.

In this section, we have given a general form for the impact parameter dependent cross section. The cross sections in Eqs. (7) and (8) are similar to the TMD factorization for photons. We see that $S_{\sigma \mu}(P, p)$ appear in Eqs. (7) and (8) as nonperturbative soft correlation functions for photons. In the next section, we will show how to handle soft correlation functions in the classical field approximation.

\section{CLASSICAL FIELD APPROXIMATION}

In this section, we take the classical field approximation for the photon field $A^{\mu}(x)$. We follow the idea in early works $[71,75,76]$ and extend their original formalism by including impact parameters $\mathbf{b}_{1 T}$ and $\mathbf{b}_{2 T}$.

We consider collisions of two nuclei in the center of mass frame as shown in Fig. 2. The fluid velocities of two nuclei are $u_{1}^{\mu}=\gamma_{1}\left(1,0,0, v_{1}\right)$ and $u_{2}^{\mu}=\gamma_{2}\left(1,0,0,-v_{2}\right)$ with $\gamma_{1,2}=1 / \sqrt{1-v_{1,2}^{2}}$ being the Lorentz factor. At very high energy, these four-velocities have properties $u_{1}^{\mu} \propto n_{+}^{\mu}$ and $u_{2}^{\mu} \propto n_{-}^{\mu}$, where $n_{+}^{\mu}$ and $n_{-}^{\mu}$ are lightlike vectors given by Eq. (B4). The charge currents are then $j_{1,2}^{\mu}=Z_{1,2} e \rho_{1,2}(\mathbf{x}) u_{1,2}^{\mu}$, where $\rho_{1,2}(\mathbf{x})$ are the charge densities. By choosing the Lorentz gauge $\partial_{\mu} A^{\mu}=0$ or $p \cdot A(p)=0$, we can solve the Maxwell equations $\partial_{\rho} \partial^{\rho} A_{(1,2)}^{\mu}=j_{1,2}^{\mu}$ to derive the classical photon (electromagnetic or EM) fields $A_{(1,2)}^{\mu}$. We introduce the nuclear form factor $F(\mathbf{k})$ as the Fourier transformation of $\rho(\mathbf{x})$

$$
\begin{aligned}
F(\mathbf{p}) & =\int d^{3} x e^{-i \mathbf{p} \cdot \mathbf{x}} \rho(\mathbf{x}), \\
\rho(\mathbf{x}) & =\int \frac{d^{3} p}{(2 \pi)^{3}} e^{i \mathbf{p} \cdot \mathbf{x}} F(\mathbf{p}) .
\end{aligned}
$$

Then the classical photon fields in momentum space are

$$
A_{(1,2)}^{\mu}(p)=2 \pi Z_{1,2} e \delta\left(p \cdot u_{1,2}\right) \frac{F(p)}{-p^{2}} u_{1,2}^{\mu}
$$

where we have replaced $\delta\left(p^{0}\right)$ by its covariant form $\delta\left(p \cdot u_{1,2}\right)$ [88]. Note that in Refs. [71,75], an extra phase factor $e^{i \mathbf{p}_{T} \cdot \mathbf{b}_{T}}$ is included in $A_{(1)}^{\mu}(p)$ to describe the dependence on the impact parameter. Since we have already introduced the dependence on impact parameters systematically into the differential cross sections (4), (7), we do not need to add this phase factor into $A_{(1,2)}^{\mu}(p)$ in (11). One can verifiy that our formalism is equivalent to Refs. [71,75].

The matrix elements of photon operators in Eq. (A18) are assumed to take the form with $i=1,2$ labeling two nuclei 


$$
\begin{aligned}
\left\langle P_{i}^{\prime}\left|A_{\sigma}^{\dagger}\left(p_{i}^{\prime}\right) A_{\mu}\left(p_{i}\right)\right| P_{i}\right\rangle & \approx A_{\sigma}^{*}\left(p_{i}^{\prime}\right) A_{\mu}\left(p_{i}\right) 2 \sqrt{\left(P_{i} \cdot u_{i}\right)\left(P_{i}^{\prime} \cdot u_{i}\right)}(2 \pi)^{3} \delta^{(3)}\left(\bar{p}_{i}-\bar{p}_{i}^{\prime}-\bar{P}_{i}+\bar{P}_{i}^{\prime}\right) \\
& =A_{\sigma}^{*}\left(p_{i}^{\prime}\right) A_{\mu}\left(p_{i}\right) \frac{1}{\gamma} 2 \sqrt{E_{P i} E_{P i^{\prime}}}(2 \pi)^{3} \delta\left(p_{i} \cdot \bar{u}_{i}-p_{i}^{\prime} \cdot \bar{u}_{i}-P_{i} \cdot \bar{u}_{i}+P_{i}^{\prime} \cdot \bar{u}_{i}\right) \delta^{(2)}\left(\mathbf{p}_{i T}-\mathbf{p}_{i T}^{\prime}-\mathbf{P}_{i T}+\mathbf{P}_{i T}^{\prime}\right)
\end{aligned}
$$

where $A_{\sigma}^{*}\left(p_{i}^{\prime}\right) \equiv A_{\sigma(i)}^{*}\left(p_{i}^{\prime}\right)$ and $A_{\mu}\left(p_{i}\right) \equiv A_{\mu(i)}\left(p_{i}\right)$ denote the classical photon fields in (11) and $\bar{a}_{i}^{\mu}$ denote the components of $a_{i}^{\mu}$ for $a=p, p^{\prime}, P, P^{\prime}$ that are perpendicular to $u_{i}^{\mu}, \bar{a}_{i} \cdot u_{i}=0$, which are given by

$$
\bar{a}_{i}^{\mu} \equiv-\left(a_{i} \cdot \bar{u}_{i}\right) \bar{u}_{i}^{\mu}+g_{T}^{\mu \nu} a_{i, \nu}=\Delta_{u_{i}}^{\mu \nu} a_{i, \nu}
$$

with $\bar{u}_{i}^{\mu} \equiv \gamma(v, 0,0,1), g_{T}^{\mu \nu} \equiv \operatorname{diag}(0,-1,-1,0)$, and $\Delta_{u_{i}}^{\mu \nu} \equiv g^{\mu \nu}-u_{i}^{\mu} u_{i}^{\nu}$. Note that $\bar{a}_{i}^{\mu}$ are actually the spatial components of $a_{i}^{\mu}$ in the comoving frame of the nucleus labeled by $i$. So $a_{i}^{\mu}$ can be decomposed as $a_{i}^{\mu}=\left(a_{i} \cdot u_{i}\right) u_{i}^{\mu}+\bar{a}_{i}^{\mu}$. The explicit forms of $\bar{a}_{i}^{\mu}$ for $a=p, p^{\prime}$ are given by $a_{i}^{\mu}$ under the conditions $a_{i} \cdot u_{i}=0$,

$$
\bar{a}_{1}^{\mu}=\left(a_{1}^{0}, \boldsymbol{a}_{1 T}, \frac{a_{1}^{0}}{v_{1}}\right), \quad \bar{a}_{2}^{\mu}=\left(a_{2}^{0}, \boldsymbol{a}_{2 T},-\frac{a_{2}^{0}}{v_{2}}\right) .
$$

Now we can derive the cross section from Eq. (A18) for collisions of two equal nuclei $\left(Z_{1}=Z_{2}=Z\right.$ and $\left.v_{1}=v_{2}=v\right)$ by using Eqs. (11) and (12) in Eq. (A18) and completing the integrals over $p_{1}^{\prime}, p_{2}^{\prime}, p_{1} \cdot u_{1}$, and $p_{2} \cdot u_{2}$. The result is

$$
\begin{aligned}
\sigma \approx & \frac{e^{4} Z^{4}}{2 \gamma^{2} v} \int d^{2} \mathbf{b}_{T} d^{2} \mathbf{b}_{1 T} d^{2} \mathbf{b}_{2 T} \int \frac{d^{3} k_{1}}{(2 \pi)^{3} 2 E_{k 1}} \frac{d^{3} k_{2}}{(2 \pi)^{3} 2 E_{k 2}} \int \frac{d^{3} \bar{p}_{1}}{(2 \pi)^{3}} \frac{d^{3} \bar{p}_{2}}{(2 \pi)^{3}} \int \frac{d^{2} \mathbf{P}_{1 T}}{(2 \pi)^{2}} \frac{d^{2} \mathbf{P}_{2 T}}{(2 \pi)^{2}} \frac{d^{2} \mathbf{P}_{1 T}^{\prime}}{(2 \pi)^{2}} \frac{d^{2} \mathbf{P}_{2 T}^{\prime}}{(2 \pi)^{2}} G^{2}\left[\left(P_{1}^{\prime z}-P_{A 1}^{z}\right)^{2}\right] \\
& \times \phi_{T}\left(\mathbf{P}_{1 T}\right) \phi_{T}\left(\mathbf{P}_{2 T}\right) \phi_{T}^{*}\left(\mathbf{P}_{1 T}^{\prime}\right) \phi_{T}^{*}\left(\mathbf{P}_{2 T}^{\prime}\right) \times e^{-i \mathbf{b}_{1 T} \cdot \mathbf{\Delta}_{1 T}} e^{-i \mathbf{b}_{2 T} \cdot \mathbf{\Delta}_{2 T}} \delta^{(2)}\left(\mathbf{b}_{T}-\mathbf{b}_{1 T}+\mathbf{b}_{2 T}\right) \frac{F\left(-\bar{p}_{1}^{2}\right)}{-\bar{p}_{1}^{2}} \frac{F\left(-\bar{p}_{2}^{2}\right)}{-\bar{p}_{2}^{2}} \frac{F^{*}\left(-\bar{p}_{1}^{\prime 2}\right)}{-\bar{p}_{1}^{\prime 2}} \frac{F^{*}\left(-\bar{p}_{2}^{\prime 2}\right)}{-\bar{p}_{2}^{\prime 2}} \\
& \times \sum_{\text {spin of } l, \bar{l}}\left[u_{1 \mu} u_{2 \nu} L^{\mu \nu}\left(\bar{p}_{1}, \bar{p}_{2} ; k_{1}, k_{2}\right)\right]\left[u_{1 \sigma} u_{2 \rho} L^{\sigma \rho *}\left(\bar{p}_{1}^{\prime}, \bar{p}_{2}^{\prime} ; k_{1}, k_{2}\right)\right](2 \pi)^{4} \delta^{(4)}\left(\bar{p}_{1}+\bar{p}_{2}-k_{1}-k_{2}\right),
\end{aligned}
$$

where $\bar{p}_{1}, \bar{p}_{2}, \bar{p}_{1}^{\prime}$, and $\bar{p}_{2}^{\prime}$ are given by Eq. (14) and $\bar{p}_{i}^{\prime}=\bar{p}_{i}-\bar{P}_{i}+\bar{P}_{i}^{\prime}$ for $i=1,2$. Note that $\bar{p}_{i}, \bar{p}_{i}^{\prime}, \bar{P}_{i}$, and $\bar{P}_{i}^{\prime}$ are momenta perpendicular to the four-velocity $u_{i}^{\mu}$ which have three independent components, while quantities with index $T$ are transverse ones perpendicular to the beam direction. We denote the 0 components of photon momenta $p_{i}$ and $p_{i}^{\prime}$ as $\omega_{i}$ and $\omega_{i}^{\prime}$ for $i=1,2$, respectively, and then the conditions $\bar{p}_{i}^{\prime}=\bar{p}_{i}-\bar{P}_{i}+\bar{P}_{i}^{\prime}$ require $\omega_{i}^{\prime}=\omega_{i}-E_{P i}+E_{P i^{\prime}}$ and $\mathbf{p}_{i T}^{\prime}=\mathbf{p}_{i T}-\mathbf{P}_{i T}+\mathbf{P}_{i T}^{\prime}$. In the derivation of (15), the four-momentum integrals are treated as $d^{4} p_{i}=d\left(p_{i} \cdot u_{i}\right) d^{3} \bar{p}_{i} \equiv$ $d\left(p_{i} \cdot u_{i}\right) d\left(-p_{i} \cdot \bar{u}_{i}\right) d^{2} \mathbf{p}_{i T}$, because of $-p_{i} \cdot \bar{u}_{i}=\omega_{i} /(v \gamma) \quad$ when applying $\quad p_{i} \cdot u_{i}=0$, we have $d^{4} p_{i}=$ $d\left(p_{i} \cdot u_{i}\right) d \omega_{i} d^{2} \mathbf{p}_{i T} /(v \gamma)$.

Completing the integrals over $\mathbf{b}_{1 T}, \mathbf{b}_{2 T}, \mathbf{b}_{T}, \mathbf{P}_{i T}$, and $\mathbf{P}_{i T}^{\prime}$ in Eq. (15), we obtain

$$
\begin{aligned}
\sigma= & \frac{e^{4} Z^{4}}{2 \gamma^{4} v^{3}} \int \frac{d^{3} k_{1}}{(2 \pi)^{3} 2 E_{k 1}} \frac{d^{3} k_{2}}{(2 \pi)^{3} 2 E_{k 2}} \int \frac{d \omega_{1} d^{2} \mathbf{p}_{1 T}}{(2 \pi)^{3}} \int \frac{d \omega_{2} d^{2} \mathbf{p}_{2 T}}{(2 \pi)^{3}} \frac{F\left(-\bar{p}_{1}^{2}\right)}{-\bar{p}_{1}^{2}} \frac{F\left(-\bar{p}_{2}^{2}\right)}{-\bar{p}_{2}^{2}} \frac{F^{*}\left(-\bar{p}_{1}^{2}\right)}{-\bar{p}_{1}^{2}} \frac{F^{*}\left(-\bar{p}_{2}^{2}\right)}{-\bar{p}_{2}^{2}} \\
& \times \sum_{\text {spin of } l, \bar{l}}\left[u_{1 \mu} u_{2 \nu} L^{\mu \nu}\left(\bar{p}_{1}, \bar{p}_{2} ; k_{1}, k_{2}\right)\right]\left[u_{1 \sigma} u_{2 \rho} L^{\sigma \rho *}\left(\bar{p}_{1}, \bar{p}_{2} ; k_{1}, k_{2}\right)\right](2 \pi)^{4} \delta^{(4)}\left(\bar{p}_{1}+\bar{p}_{2}-k_{1}-k_{2}\right),
\end{aligned}
$$

where we have used $P_{1}^{\prime z}=P_{A 1}^{z}$ as the solution to the energy conservation equation with the conditions $\mathbf{P}_{1 T}=\mathbf{P}_{1 T}^{\prime}$ and $\mathbf{P}_{2 T}=\mathbf{P}_{2 T}^{\prime}$. The result of (16) is consistent with the results in Refs. [71,75]. 
On the other hand, we can carry out the integrals over $p_{i}^{\prime} \cdot u_{i}, p_{i} \cdot u_{i}, \omega_{i}^{\prime}$ and $\mathbf{P}_{i T}^{\prime}$ for $i=1,2$ in Eq. (A18), and the result is

$$
\begin{aligned}
\sigma= & \frac{Z^{4} e^{4}}{2 \gamma^{4} v^{3}} \int d^{2} \mathbf{b}_{T} d^{2} \mathbf{b}_{1 T} d^{2} \mathbf{b}_{2 T} \int \frac{d \omega_{1} d^{2} \mathbf{p}_{1 T}}{(2 \pi)^{3}} \frac{d \omega_{2} d^{2} \mathbf{p}_{2 T}}{(2 \pi)^{3}} \int \frac{d^{2} \mathbf{P}_{1 T}}{(2 \pi)^{2}} \frac{d^{2} \mathbf{P}_{2 T}}{(2 \pi)^{2}} \phi_{T}\left(\mathbf{P}_{1 T}\right) \phi_{T}\left(\mathbf{P}_{2 T}\right) \delta^{(2)}\left(\mathbf{b}_{T}-\mathbf{b}_{1 T}+\mathbf{b}_{2 T}\right) \\
& \times \int \frac{d^{2} \mathbf{p}_{1 T}^{\prime}}{(2 \pi)^{2}} e^{-i \mathbf{b}_{1 T} \cdot\left(\mathbf{p}_{1 T}^{\prime}-\mathbf{p}_{1 T}\right)} \frac{F^{*}\left(-\bar{p}_{1}^{\prime 2}\right)}{-\bar{p}_{1}^{\prime 2}} \frac{F\left(-\bar{p}_{1}^{2}\right)}{-\bar{p}_{1}^{2}} \boldsymbol{\phi}_{T}^{*}\left(\mathbf{P}_{1 T}^{\prime}\right) \\
& \times \int \frac{d^{2} \mathbf{p}_{2 T}^{\prime}}{(2 \pi)^{2}} e^{-i \mathbf{b}_{2 T} \cdot\left(\mathbf{p}_{2 T}^{\prime}-\mathbf{p}_{2 T}\right)} \frac{F^{*}\left(-\bar{p}_{2}^{\prime 2}\right)}{-\bar{p}_{2}^{\prime 2}} \frac{F\left(-\bar{p}_{2}^{2}\right)}{-\bar{p}_{2}^{2}} \boldsymbol{\phi}_{T}^{*}\left(\mathbf{P}_{2 T}^{\prime}\right) G^{2}\left[\left(P_{1}^{\prime z}-P_{A 1}^{z}\right)^{2}\right] \\
& \times \int \frac{d^{3} k_{1}}{(2 \pi)^{3} 2 E_{k 1}} \frac{d^{3} k_{2}}{(2 \pi)^{3} 2 E_{k 2}} \sum_{\text {spin of } l, \bar{l}}\left[u_{1 \mu} u_{2 \nu} L^{\mu \nu}\left(\bar{p}_{1}, \bar{p}_{2} ; k_{1}, k_{2}\right)\right]\left[u_{1 \sigma} u_{2 \rho} L^{\sigma \rho *}\left(\bar{p}_{1}^{\prime}, \bar{p}_{2}^{\prime} ; k_{1}, k_{2}\right)\right](2 \pi)^{4} \delta^{(4)}\left(\bar{p}_{1}+\bar{p}_{2}-k_{1}-k_{2}\right),
\end{aligned}
$$

where $\omega_{i}^{\prime}=\omega_{i}-E_{P i}+E_{P i^{\prime}}, \mathbf{P}_{1 T}^{\prime}=\mathbf{P}_{1 T}-\mathbf{p}_{1 T}+\mathbf{p}_{1 T}^{\prime}$, and $\mathbf{P}_{2 T}^{\prime}=\mathbf{P}_{2 T}-\mathbf{p}_{2 T}+\mathbf{p}_{2 T}^{\prime}$. Note that $\mathbf{p}_{1 T}^{\prime}$ and $\mathbf{p}_{2 T}^{\prime}$ are now free variables. We see in the above formula that the couplings of impact parameters with nuclear momenta have been converted to those with photon momenta by integration over nuclear transverse momenta $\mathbf{P}_{1 T}^{\prime}$ and $\mathbf{P}_{2 T}^{\prime}$. Completing the integrals over $\mathbf{b}_{1 T}$ and $\mathbf{b}_{2 T}$ will give a term

$$
e^{-i \mathbf{b}_{T} \cdot\left(\mathbf{p}_{1 T}^{\prime}-\mathbf{p}_{1 T}\right)} \delta^{(2)}\left(\mathbf{p}_{1 T}+\mathbf{p}_{2 T}+\mathbf{p}_{1 T}^{\prime}-\mathbf{p}_{2 T}^{\prime}\right)
$$

in the integrand.

If we make a similar ansatz to (A9) for transverse momentum amplitudes we can simplify Eq. (17) significantly,

$$
\begin{aligned}
\phi_{T}\left(\mathbf{P}_{1 T}\right) \phi_{T}^{*}\left(\mathbf{P}_{1 T}^{\prime}\right) \phi_{T}\left(\mathbf{P}_{2 T}\right) \phi_{T}^{*}\left(\mathbf{P}_{2 T}^{\prime}\right) \approx & (2 \pi)^{4} \delta^{(2)}\left[\left(\mathbf{P}_{1 T}+\mathbf{P}_{1 T}^{\prime}\right) / 2\right] \delta^{(2)}\left[\left(\mathbf{P}_{2 T}+\mathbf{P}_{2 T}^{\prime}\right) / 2\right] G_{T}\left[\left(\mathbf{P}_{1 T}^{\prime}-\mathbf{P}_{1 T}\right)^{2}\right] G_{T}\left[\left(\mathbf{P}_{2 T}^{\prime}-\mathbf{P}_{2 T}\right)^{2}\right] \\
= & (2 \pi)^{4} \delta^{(2)}\left[\mathbf{P}_{1 T}-\left(\mathbf{p}_{1 T}-\mathbf{p}_{1 T}^{\prime}\right) / 2\right] \delta^{(2)}\left[\mathbf{P}_{2 T}-\left(\mathbf{p}_{2 T}-\mathbf{p}_{2 T}^{\prime}\right) / 2\right] \\
& \times G_{T}\left[\left(\mathbf{p}_{1 T}^{\prime}-\mathbf{p}_{1 T}\right)^{2}\right] G_{T}\left[\left(\mathbf{p}_{2 T}^{\prime}-\mathbf{p}_{2 T}\right)^{2}\right]
\end{aligned}
$$

where $G_{T}\left(x^{2}\right)$ may differ from $G\left(x^{2}\right)$ for longitudinal momenta but with similar behavior: it is a positive function with $G_{T}(0)=1$ and decreases rapidly with growing $x^{2}$. Then the integrals over $\mathbf{P}_{1 T}$ and $\mathbf{P}_{2 T}$ in Eq. (17) can be completed, and we obtain

$$
\begin{aligned}
\sigma= & \frac{Z^{4} e^{4}}{2 \gamma^{4} v^{3}} \int d^{2} \mathbf{b}_{T} d^{2} \mathbf{b}_{1 T} d^{2} \mathbf{b}_{2 T} \delta^{(2)}\left(\mathbf{b}_{T}-\mathbf{b}_{1 T}+\mathbf{b}_{2 T}\right) \int \frac{d \omega_{1} d^{2} \mathbf{p}_{1 T}}{(2 \pi)^{3}} \frac{d \omega_{2} d^{2} \mathbf{p}_{2 T}}{(2 \pi)^{3}} \\
& \times \int \frac{d^{2} \mathbf{p}_{1 T}^{\prime}}{(2 \pi)^{2}} e^{-i \mathbf{b}_{1 T} \cdot\left(\mathbf{p}_{1 T}^{\prime}-\mathbf{p}_{1 T}\right)} \frac{F^{*}\left(-\bar{p}_{1}^{\prime 2}\right)}{-\bar{p}_{1}^{\prime 2}} \frac{F\left(-\bar{p}_{1}^{2}\right)}{-\bar{p}_{1}^{2}} G_{T}\left[\left(\mathbf{p}_{1 T}^{\prime}-\mathbf{p}_{1 T}\right)^{2}\right] \\
& \times \int \frac{d^{2} \mathbf{p}_{2 T}^{\prime}}{(2 \pi)^{2}} e^{-i \mathbf{b}_{2 T} \cdot\left(\mathbf{p}_{2 T}^{\prime}-\mathbf{p}_{2 T}\right)} \frac{F^{*}\left(-\bar{p}_{2}^{\prime 2}\right)}{-\bar{p}_{2}^{\prime 2}} \frac{F\left(-\bar{p}_{2}^{2}\right)}{-\bar{p}_{2}^{2}} G_{T}\left[\left(\mathbf{p}_{2 T}^{\prime}-\mathbf{p}_{2 T}\right)^{2}\right] \\
& \times \int \frac{d^{3} k_{1}}{(2 \pi)^{3} 2 E_{k 1}} \frac{d^{3} k_{2}}{(2 \pi)^{3} 2 E_{k 2}} \sum_{\text {spin of } l, \bar{l}}\left[u_{1 \mu} u_{2 \nu} L^{\mu \nu}\left(\bar{p}_{1}, \bar{p}_{2} ; k_{1}, k_{2}\right)\right]\left[u_{1 \sigma} u_{2 \rho} L^{\sigma \rho *}\left(\bar{p}_{1}^{\prime}, \bar{p}_{2}^{\prime} ; k_{1}, k_{2}\right)\right](2 \pi)^{4} \delta^{(4)}\left(\bar{p}_{1}+\bar{p}_{2}-k_{1}-k_{2}\right),
\end{aligned}
$$

where we have used $P_{1}^{z z}=P_{A 1}^{z}$ as the solution to the energy conservation equation with the conditions $\mathbf{P}_{1 T}=-\mathbf{P}_{1 T}^{\prime}$ and $\mathbf{P}_{2 T}=-\mathbf{P}_{2 T}^{\prime}$, and $\omega_{i}^{\prime}=\omega_{i}$ for $i=1,2$.

We emphasize that one of our main results in this work is the cross section (20) with impact parameter dependence which encodes the information of photons in transverse phase space. This is the basis for the derivation of EPA results in Sec. IV at the relativistic limit. We will show that Eq. (20) contains all high order contributions of $\gamma \gamma \rightarrow l \bar{l}$ at the tree level. In Sec. VI, we will compute cross sections based on Eq. (20) and compare our results with experimental data. 
We will also parametrize the TMD correlation function $S_{\mu \nu}$ in Eq. (9) and implement the TMD factorization formalism in Sec. V.

\section{CONNECTION TO EPA}

In this section, we will derive the EPA result from Eq. (20). Now we evaluate $u_{1 \mu} u_{2 \nu} L^{\mu \nu}\left(\bar{p}_{1}, \bar{p}_{2} ; k_{1}, k_{2}\right)$ and $u_{1 \sigma} u_{2 \rho} L^{\sigma \rho *}\left(\bar{p}_{1}^{\prime}, \bar{p}_{2}^{\prime} ; k_{1}, k_{2}\right)$ in Eq. (17). In order to simplify notations, from now on, we resume the use of $p_{1}, p_{2}, p_{1}^{\prime}$, and $p_{2}^{\prime}$ in $L^{\mu \nu}$ and $L^{\sigma \rho *}$ for $\bar{p}_{1}, \bar{p}_{2}, \bar{p}_{1}^{\prime}$, and $\bar{p}_{2}^{\prime}$, respectively, if there is no ambiguity. It is convenient to rewrite $u_{1 \mu} u_{2 \nu} \Gamma^{\mu \nu}\left(p_{1}, p_{2} ; k_{1} k_{2}\right)$ in light-cone coordinates,

$$
\begin{aligned}
u_{1 \mu} u_{2 \nu} L^{\mu \nu} & \left(p_{1}, p_{2} ; k_{1} k_{2}\right) \\
= & \gamma^{2} v^{2} \frac{p_{1}^{i}}{\omega_{1}} \frac{p_{2}^{j}}{\omega_{2}} L^{i j}-2 \gamma^{2} v^{2}\left(\frac{p_{1}^{i}}{\omega_{1}} \frac{p_{2}^{+}}{\omega_{2}} L^{i-}+\frac{p_{1}^{-}}{\omega_{1}} \frac{p_{2}^{j}}{\omega_{2}} L^{+j}\right) \\
& +4 \gamma^{2} v^{2} \frac{p_{1}^{-}}{\omega_{1}} \frac{p_{2}^{+}}{\omega_{2}} L^{+-},
\end{aligned}
$$

where $i, j=x, y$ stand for transverse directions, we have used Eq. (A23), and $p_{1,2}^{ \pm}$are given by

$$
p_{1}^{ \pm}=\frac{\omega_{1}}{\sqrt{2}}\left(1 \pm \frac{1}{v}\right), \quad p_{2}^{ \pm}=\frac{\omega_{2}}{\sqrt{2}}\left(1 \mp \frac{1}{v}\right) .
$$

At the relativistic limit $v \rightarrow 1$ and $\gamma \rightarrow \infty$, the following power counting rules hold in the light-cone coordinate

$$
\begin{array}{llll}
\frac{p_{1}^{+}}{\omega_{1}}, & \frac{p_{2}^{-}}{\omega_{2}} \sim \mathcal{O}(1), & \frac{p_{1}^{-}}{\omega_{1}}, & \frac{p_{2}^{+}}{\omega_{2}} \sim \mathcal{O}\left(\gamma^{-2}\right), \\
\frac{\mathbf{p}_{1}^{T}}{\omega_{1}}, & \frac{\mathbf{p}_{2}^{T}}{\omega_{2}} \sim \mathcal{O}\left(\gamma^{-1}\right) . &
\end{array}
$$

It implies that photons are almost on-shell [71],

$$
\frac{p^{2}}{\omega^{2}} \sim \mathcal{O}\left(\gamma^{-2}\right)
$$

Following (23), the first, second, and third terms of (21) are $O(1), O\left(\gamma^{-1}\right)$, and $O\left(\gamma^{-2}\right)$, respectively. Therefore the leading order contribution comes from the first term. The expansion in (21) can also be interpreted as the photon virtuality expansion. Details can be found in Appendix B.

At the relativistic limit, the cross section (20) can be put into a compact form

$$
\sigma=\sigma_{0}+\delta \sigma
$$

where $\sigma_{0}$ is the leading order contribution from the first term of Eq. (21)

$$
\begin{gathered}
\gamma^{2} v^{2} \frac{p_{1}^{i} p_{2}^{j}}{\omega_{1} \omega_{2}} L^{i j} \approx \gamma^{2} v^{2} \frac{\left|\mathbf{p}_{1 T}\right|\left|\mathbf{p}_{2 T}\right|}{\omega_{1} \omega_{2}} \hat{p}_{1}^{i} \hat{p}_{2}^{j} L^{i j}\left(p_{1}, p_{2} ; k_{1}, k_{2}\right), \\
\gamma^{2} v^{2} \frac{p_{1}^{\prime k} p_{2}^{\prime l}}{\omega_{1}^{\prime} \omega_{2}^{\prime}} L^{k l} \approx \gamma^{2} v^{2} \frac{\left|\mathbf{p}_{1 T}^{\prime}\right|\left|\mathbf{p}_{2 T}^{\prime}\right|}{\omega_{1}^{\prime} \omega_{2}^{\prime}} \hat{p}_{1}^{\prime k} \hat{p}_{2}^{\prime l} L^{k l}\left(p_{1}^{\prime}, p_{2}^{\prime} ; k_{1}, k_{2}\right),
\end{gathered}
$$

and $\delta \sigma$ represents the corrections from other terms. In the above formula, $i, j, k, l$ are indices of two transverse directions, and $\hat{p}_{1,2}^{i}$ and $\hat{p}_{1,2}^{i}$ denote the directions (unit vectors) of $\mathbf{p}_{1 T, 2 T}$ and $\mathbf{p}_{1 T, 2 T}^{\prime}$, respectively, which play the role of polarization vectors of photons so the summation over $i, j, k, l$ represents that over photon polarizations. Therefore we can define the last two lines of Eq. (20) as a kind of cross section of the photon fusion to produce dileptons,

$$
\begin{aligned}
\sigma_{\gamma \gamma \rightarrow \bar{l}}\left(p_{1}, p_{2} ; p_{1}^{\prime}, p_{2}^{\prime}\right)= & \frac{1}{8 \omega_{1}^{\prime} \omega_{2}^{\prime}} \int \frac{d^{3} k_{1}}{(2 \pi)^{3} 2 E_{k 1}} \frac{d^{3} k_{2}}{(2 \pi)^{3} 2 E_{k 2}} \sum_{\text {spin of } l, \bar{l}}\left[\hat{p}_{1}^{i} \hat{p}_{2}^{j} L^{i j}\left(p_{1}, p_{2} ; k_{1}, k_{2}\right)\right] \\
& \times\left[\hat{p}_{1}^{\prime k} \hat{p}_{2}^{\prime l} L^{k l}\left(p_{1}^{\prime}, p_{2}^{\prime} ; k_{1}, k_{2}\right)\right](2 \pi)^{4} \delta^{(4)}\left(p_{1}+p_{2}-k_{1}-k_{2}\right) .
\end{aligned}
$$

When $p_{1}=p_{1}^{\prime}$ and $p_{2}=p_{2}^{\prime}, \sigma_{\gamma \gamma \rightarrow \bar{l}}\left(p_{1} p_{2}, p_{1}^{\prime} p_{2}^{\prime}\right)$ becomes the ordinary cross section for production of lepton pairs by two photons.

The leading order cross section from Eq. (20) is put into the form

$$
\begin{aligned}
\sigma_{0}\left(A_{1} A_{2} \rightarrow \bar{l}\right) \approx & \int d^{2} \mathbf{b}_{T} d^{2} \mathbf{b}_{1 T} d^{2} \mathbf{b}_{2 T} \delta^{(2)}\left(\mathbf{b}_{T}-\mathbf{b}_{1 T}+\mathbf{b}_{2 T}\right) \\
& \times \int d \omega_{1} d^{2} \mathbf{p}_{1 T} d \omega_{2} d^{2} \mathbf{p}_{2 T} \frac{Z^{2} \alpha}{\omega_{1} \pi^{2}} \int \frac{d^{2} \mathbf{p}_{1 T}^{\prime}}{(2 \pi)^{2}} e^{-i \mathbf{b}_{1 T} \cdot\left(\mathbf{p}_{1 T}^{\prime}-\mathbf{p}_{1 T}\right)}\left|\mathbf{p}_{1 T}\right|\left|\mathbf{p}_{1 T}^{\prime}\right| \frac{F^{*}\left(-p_{1}^{\prime 2}\right)}{-p_{1}^{\prime 2}} \frac{F\left(-p_{1}^{2}\right)}{-p_{1}^{2}} G_{T}\left[\left(\mathbf{p}_{1 T}^{\prime}-\mathbf{p}_{1 T}\right)^{2}\right] \\
& \times \frac{Z^{2} \alpha}{\omega_{1} \pi^{2}} \int \frac{d^{2} \mathbf{p}_{2 T}^{\prime}}{(2 \pi)^{2}} e^{-i \mathbf{b}_{2 T} \cdot\left(\mathbf{p}_{2 T}^{\prime}-\mathbf{p}_{2 T}\right)}\left|\mathbf{p}_{2 T}\right|\left|\mathbf{p}_{2 T}^{\prime}\right| \frac{F^{*}\left(-p_{2}^{\prime 2}\right)}{-p_{2}^{\prime 2}} \frac{F\left(-p_{2}^{2}\right)}{-p_{2}^{2}} G_{T}\left[\left(\mathbf{p}_{2 T}^{\prime}-\mathbf{p}_{2 T}\right)^{2}\right] \sigma_{\gamma \gamma \rightarrow l \bar{l}}\left(p_{1}, p_{2} ; p_{1}^{\prime}, p_{2}^{\prime}\right) .
\end{aligned}
$$


If we approximate $\mathbf{p}_{i T}^{\prime} \approx \mathbf{p}_{i T}$ for $i=1,2$, we have

$$
\begin{aligned}
G_{T}\left[\left(\mathbf{p}_{i T}^{\prime}-\mathbf{p}_{i T}\right)^{2}\right] & \approx 1 \\
\sigma_{\gamma \gamma \rightarrow \bar{l}}\left(p_{1}, p_{2} ; p_{1}^{\prime}, p_{2}^{\prime}\right) & \approx \sigma_{\gamma \gamma \rightarrow \bar{l}}\left(p_{1}, p_{2}\right) \\
& \approx \sigma_{\gamma \gamma \rightarrow \bar{l}}\left(\omega_{1}, \omega_{2}\right) .
\end{aligned}
$$

Then Eq. (28) can be written in a compact way

$$
\begin{aligned}
\sigma_{0}\left(A_{1} A_{2} \rightarrow \bar{l}\right) \approx & \int d^{2} \mathbf{b}_{T} d^{2} \mathbf{b}_{1 T} d^{2} \mathbf{b}_{2 T} \int d \omega_{1} d^{2} \mathbf{p}_{1 T} d \omega_{2} d^{2} \mathbf{p}_{2 T} n_{A 1}\left(\omega_{1}, \mathbf{b}_{1 T}, \mathbf{p}_{1 T}\right) n_{A 2}\left(\omega_{2}, \mathbf{b}_{2 T}, \mathbf{p}_{2 T}\right) \\
& \times \delta^{(2)}\left(\mathbf{b}_{T}-\mathbf{b}_{1 T}+\mathbf{b}_{2 T}\right) \sigma_{\gamma \gamma \rightarrow \bar{l}}\left(\omega_{1}, \omega_{2}\right) \\
= & \int d^{2} \mathbf{b}_{T} d^{2} \mathbf{b}_{1 T} d^{2} \mathbf{b}_{2 T} \int d \omega_{1} d \omega_{2} n_{A 1}\left(\omega_{1}, \mathbf{b}_{1 T}\right) n_{A 2}\left(\omega_{2}, \mathbf{b}_{2 T}\right) \delta^{(2)}\left(\mathbf{b}_{T}-\mathbf{b}_{1 T}+\mathbf{b}_{2 T}\right) \sigma_{\gamma \gamma \rightarrow \bar{l}}\left(\omega_{1}, \omega_{2}\right),
\end{aligned}
$$

where the photon flux spectra from $A_{1}$ and $A_{2}$ are

$$
\begin{aligned}
n_{A i}\left(\omega_{i}, \mathbf{b}_{i T}, \mathbf{p}_{i T}\right) \approx & \frac{Z^{2} \alpha}{\omega_{i} \pi^{2}} \int \frac{d^{2} \mathbf{p}_{i T}^{\prime}}{(2 \pi)^{2}}\left|\mathbf{p}_{i T}\right|\left|\mathbf{p}_{i T}^{\prime}\right| e^{-i \mathbf{b}_{i T} \cdot\left(\mathbf{p}_{i T}^{\prime}-\mathbf{p}_{i T}\right)} \\
& \times \frac{F^{*}\left(-p_{i}^{\prime 2}\right)}{-p_{i}^{\prime 2}} \frac{F\left(-p_{i}^{2}\right)}{-p_{i}^{2}}, \\
n_{A i}\left(\omega_{i}, \mathbf{b}_{i T}\right)= & \int d^{2} \mathbf{p}_{i T} n_{A i}\left(\omega_{i}, \mathbf{b}_{i T}, \mathbf{p}_{i T}\right) \\
= & \frac{4 Z^{2} \alpha}{\omega_{i}}\left|\int \frac{d^{2} \mathbf{p}_{i T}}{(2 \pi)^{2}} e^{i \mathbf{b}_{i T} \cdot \mathbf{p}_{i T}} \mathbf{p}_{i T} \frac{F\left(-p_{i}^{2}\right)}{-p_{i}^{2}}\right|^{2},
\end{aligned}
$$

for $i=1,2$. The impact parameter structure in Eq. (30) is similar to the space-dependent photon flux defined in Refs. $[85,86,89]$. The approximation that leads to the result of (30) is called the generalized EPA or gEPA [71].
We can complete the integrals over $\mathbf{b}_{T}, \mathbf{b}_{1 T}$, and $\mathbf{b}_{2 T}$ in Eq. (30) to obtain

$$
\sigma_{0}\left(A_{1} A_{2} \rightarrow \bar{l}\right)=\int d \omega_{1} d \omega_{2} n_{A 1}\left(\omega_{1}\right) n_{A 2}\left(\omega_{2}\right) \sigma_{\gamma \gamma \rightarrow \bar{l}}\left(\omega_{1}, \omega_{2}\right)
$$

where $n_{A 1}$ and $n_{A 2}$ are the photon fluxes generated by $A_{1}$ and $A_{2}$, respectively, and are given by

$$
n_{A i}\left(\omega_{i}\right)=\frac{4 Z^{2} \alpha}{\omega_{i}} \int \frac{d^{2} \mathbf{p}_{i T}}{(2 \pi)^{2}} \mathbf{p}_{i T}^{2}\left|\frac{F\left(-p_{i}^{2}\right)}{-p_{i}^{2}}\right|^{2}
$$

for $i=1,2$. Note that the photons are very close to real ones as shown in Eq. (24). The approximation that leads to the result of (32) is called EPA.

About $\delta \sigma$ in (25), the cross section beyond the EPA, with the same approximation as for Eq. (30), we obtain

$$
\delta \sigma=\frac{Z^{4} \alpha^{2} v}{8 \pi^{4}} \int \frac{d^{3} k_{1}}{(2 \pi)^{3} 2 E_{k_{1}}} \frac{d^{3} k_{2}}{(2 \pi)^{3} 2 E_{k_{2}}} \int \frac{d \omega_{1}}{\omega_{1}^{2}} \frac{d \omega_{2}}{\omega_{2}^{2}} \int d^{2} \mathbf{p}_{1 T} d^{2} \mathbf{p}_{2 T}\left|\frac{F\left(-p_{1}^{2}\right)}{-p_{1}^{2}}\right|^{2}\left|\frac{F\left(-p_{2}^{2}\right)}{-p_{2}^{2}}\right|^{2}(2 \pi)^{4} \delta^{4}\left(p_{1}+p_{2}-k_{1}-k_{2}\right) \mathcal{I},
$$

where $\mathcal{I}$ is defined through

$$
u_{1 \mu} u_{2 \nu} L^{\mu \nu} u_{1 \sigma} u_{2 \rho} L^{\sigma \rho *}=\frac{\gamma^{4} v^{4}}{\omega_{1}^{2} \omega_{2}^{2}}\left[p_{1}^{i} p_{2}^{j} p_{1}^{k} p_{2}^{l} L^{i j} L^{\sigma \rho *}+\mathcal{I}\right]
$$

where $L^{\mu \nu} \equiv L^{\mu \nu}\left(p_{1}, p_{2} ; k_{1}, k_{2}\right)$ and $L^{\sigma \rho *} \equiv\left(p_{1}, p_{2} ; k_{1}, k_{2}\right)$ which are given by (A22). Note that the first term inside the square brackets gives $\sigma_{0}$. The explicit form of $\mathcal{I}$ is given by

$$
\begin{aligned}
\mathcal{I}= & -2 p_{1}^{i} p_{2}^{j} L^{i j}\left(p_{2}^{+} p_{1}^{k} L^{k-*}+p_{1}^{-} p_{2}^{k} L^{+k *}\right)+4 p_{1}^{i} p_{2}^{j} p_{1}^{-} p_{2}^{+} L^{i j} L^{+-*}-2\left(p_{2}^{+} p_{1}^{i} L^{i-}+p_{1}^{-} p_{2}^{i} L^{+i}\right) p_{1}^{k} p_{2}^{l} L^{k l *} \\
& +4\left(p_{2}^{+} p_{1}^{i} L^{i-}+p_{1}^{-} p_{2}^{j} L^{+j}\right)\left(p_{2}^{+} p_{1}^{k} L^{k-*}+p_{1}^{-} p_{2}^{k} L^{+k *}\right)-8\left(p_{2}^{+} p_{1}^{i} L^{i-}+p_{1}^{-} p_{2}^{j} L^{+j}\right) p_{1}^{-} p_{2}^{+} L^{+-*} \\
& +4 p_{1}^{-} p_{2}^{+} p_{1}^{k} p_{2}^{l} L^{k l *} L^{+-}-8 p_{1}^{-} p_{2}^{+} L^{+-}\left(p_{2}^{+} p_{1}^{k} L^{k-*}+p_{1}^{-} p_{2}^{k} L^{+k *}\right)+16\left(p_{1}^{-}\right)^{2}\left(p_{2}^{+}\right)^{2} L^{+-} L^{+-*} .
\end{aligned}
$$

So we see that the cross section (17) contains not only the result of EPA but also the result beyond the EPA. 


\section{CONNECTION TO TMD FACTORIZATION FORMALISM}

In this section, we will discuss the connection of our results to those from the TMD factorization formalism. Here we just give a concise comparison between the results from our approach and those from the TMD factorization formalism. The details of the TMD factorization formalism applied to UPC can be found in Refs. [80,81,87,90]. The recent developments in the impact-parameter dependent cross sections in terms of photon Wigner functions are given in Refs. [85,86].

We work in the light-front formalism and discuss photon Wigner functions based on Refs. [85,86]. The gauge invariant TMD correlation function of EM fields [91] for an unpolarized nucleus moving in $+z$ direction is defined as

$\mathcal{W}^{\mu \nu ; \rho \sigma}\left(P_{1}, p_{1}\right)=\int \frac{d^{4} \xi}{(2 \pi)^{4}} e^{i p_{1} \cdot \xi}\left\langle P_{1}\left|F^{\mu \nu}(0) F^{\rho \sigma}(\xi)\right| P_{1}\right\rangle$.

Similarly one can define the TMD correlation function $\mathcal{W}^{\mu \nu ; \rho \sigma}\left(P_{2}, p_{2}\right)$ for the nucleus moving in $-z$ direction. In Sec. III we use the Lorentz gauge. Both $\mathcal{W}^{\mu \nu ; \rho \sigma}$ and the cross section are gauge invariant; therefore, in this section we follow the standard TMD factorization formalism to choose the light-cone gauge, $A^{ \pm}=0$, for nuclei 1 and 2 moving in the $\pm z$ direction. In the light-cone gauge the classical solutions to EM fields read

$$
A_{(1,2)}^{\mu}(p)=2 \pi Z_{1,2} e \delta\left(p \cdot u_{1,2}\right) \frac{F\left(-p^{2}\right)}{-p^{2}}\left[u_{1,2}^{\mu}-\frac{p^{\mu}}{p^{ \pm}} u_{1,2}^{ \pm}\right]
$$

where the upper/lower sign corresponds to nuclei 1 and 2 moving in the $\pm z$ direction, as a comparison, the result in the Lorentz gauge is given in Eq. (11). One can verify that the TMD correlation functions are related to the correlation functions in Eq. (9) as

$$
\begin{aligned}
& \mathcal{W}^{+\nu ;+\sigma}\left(P_{1}, p_{1}\right)=\left(p_{1}^{+}\right)^{2} S^{\nu \sigma}\left(P_{1}, p_{1}\right), \\
& \mathcal{W}^{+\nu ;+\sigma}\left(P_{2}, p_{2}\right)=\left(p_{2}^{-}\right)^{2} S^{\nu \sigma}\left(P_{2}, p_{2}\right) .
\end{aligned}
$$

Note that there is a different sign from Ref. [81].

We follow the twist expansion in the TMD factorization formalism [91-98], where the twist-2 contribution comes from $\mathcal{W}^{+i ;+j}$, the twist-3 contribution comes from $\mathcal{W}^{+i ;+-}$ and $\mathcal{W}^{i j ; l+}$, and $\mathcal{W}^{+-;+-}$contributes at twist-4.

In the order of twist-2, we can parametrize correlation functions for nuclei 1 and 2 of the same species as

$$
\begin{aligned}
& \int d p_{1}^{-} \mathcal{W}^{+i ;+j}\left(P_{1}, p_{1}\right)=\frac{1}{2} x_{1} P_{1}^{+}\left[-g_{T}^{i j} f^{\gamma}\left(x_{1}, \mathbf{p}_{1 T}^{2}\right)+\left(\frac{p_{1 T}^{i} p_{1 T}^{j}}{M^{2}}+\frac{\mathbf{p}_{1 T}^{2}}{2 M^{2}} g_{T}^{i j}\right) h_{T}^{\gamma}\left(x_{1}, \mathbf{p}_{1 T}^{2}\right)\right], \\
& \int d p_{2}^{+} \mathcal{W}^{+i ;+j}\left(P_{2}, p_{2}\right)=\frac{1}{2} x_{2} P_{2}^{-}\left[-g_{T}^{i j} f^{\gamma}\left(x_{2}, \mathbf{p}_{2 T}^{2}\right)+\left(\frac{p_{2 T}^{i} p_{2 T}^{j}}{M^{2}}+\frac{\mathbf{p}_{2 T}^{2}}{2 M^{2}} g_{T}^{i j}\right) h_{T}^{\gamma}\left(x_{2}, \mathbf{p}_{2 T}^{2}\right)\right],
\end{aligned}
$$

where $x_{1}=p_{1}^{+} / P_{1}^{+}$and $x_{2}=p_{2}^{-} / P_{2}^{-}$denote light-cone momentum fractions. The TMD distribution $f^{\gamma}\left(x_{1}, \mathbf{p}_{1 T}^{2}\right)$ represents the usual unpolarized photon distribution, while $h_{T}^{\gamma}\left(x_{1}, \mathbf{p}_{1 T}^{2}\right)$ represents the distribution function of linearly polarized photons in an unpolarized nucleus [91]. The cross section (8) up to twist-2 can be expressed by

$$
\begin{aligned}
\sigma_{\text {twist } 2} \approx & \frac{1}{32(2 \pi)^{6}} \int d^{3} k_{1} d^{3} k_{2} \int d p_{1}^{+} d^{2} \mathbf{p}_{1 T} d p_{2}^{-} d^{2} \mathbf{p}_{2 T} \frac{1}{v E_{A 1} E_{A 2} E_{k 1} E_{k 2}} \frac{1}{2 x_{1} P_{1}^{+}} \frac{p_{1 T}^{\sigma} p_{1 T}^{\mu}}{M^{2}} h_{T}^{\gamma}\left(x_{1}, \mathbf{p}_{1 T}^{2}\right) \\
& \times \frac{1}{2 x_{2} P_{2}^{-}} \frac{p_{2 T}^{\rho} p_{2 T}^{\nu}}{M^{2}} h_{T}^{\gamma}\left(x_{2}, \mathbf{p}_{2 T}^{2}\right) L_{\mu \nu ; \sigma \rho}\left(p_{1}, p_{2} ; p_{1}, p_{2} ; k_{1}, k_{2}\right)(2 \pi)^{4} \delta^{4}\left(p_{1}+p_{2}-k_{1}-k_{2}\right),
\end{aligned}
$$

where we have assumed $E_{P 1} \approx E_{A 1}$ and $E_{P 2} \approx E_{A 2}$ and performed the integrals over $\mathbf{P}_{1 T}$ and $\mathbf{P}_{2 T}$.

The TMD distributions $f^{\gamma}\left(x, \mathbf{p}_{T}^{2}\right)$ and $h_{T}^{\gamma}\left(x, \mathbf{p}_{T}^{2}\right)$ are nonperturbative in nature and cannot be derived directly from the perturbation theory. In the classical field approximation based on the classical solution (38) we obtain

$$
x f^{\gamma}\left(x, \mathbf{p}_{T}^{2}\right)=\frac{Z^{2} \alpha}{\pi^{2}} \mathbf{p}_{T}^{2}\left|\frac{F\left(-p^{2}\right)}{-p^{2}}\right|^{2}, \quad x h_{T}^{\gamma}\left(x, \mathbf{p}_{T}^{2}\right)=\frac{2 Z^{2} \alpha}{\pi^{2}} M^{2}\left|\frac{F\left(-p^{2}\right)}{-p^{2}}\right|^{2},
$$

which are consistent with the photon flux $n_{A}\left(\omega, \mathbf{p}_{T}\right)$ in Eqs. (33). The details for the derivation of the above result can be found in Appendix C. Inserting Eq. (42) into Eq. (41) yields 


$$
\begin{aligned}
\frac{d \sigma_{\text {twist } 2}}{d^{3} k_{1} d^{3} k_{2}}= & \frac{1}{2(2 \pi)^{10}} Z^{4} \alpha^{2} v \int d \omega_{1} d^{2} \mathbf{p}_{1 T} d \omega_{2} d^{2} \mathbf{p}_{2 T} \frac{1}{E_{k 1} E_{k 2}} \frac{p_{1 T}^{\sigma} p_{1 T}^{\mu} p_{2 T}^{\rho} p_{2 T}^{\nu}}{\omega_{1}^{2} \omega_{2}^{2}}\left|\frac{F\left(-p_{1}^{2}\right)}{-p_{1}^{2}}\right|^{2}\left|\frac{F\left(-p_{2}^{2}\right)}{-p_{2}^{2}}\right|^{2} \\
& \times L_{\mu \nu ; \sigma \rho}\left(p_{1}, p_{2} ; p_{1}, p_{2} ; k_{1}, k_{2}\right)(2 \pi)^{4} \delta^{4}\left(p_{1}+p_{2}-k_{1}-k_{2}\right) .
\end{aligned}
$$

It is straightforward to prove that the above result is equivalent to Eq. (30).

In the classical field approximation, the twist-3 correlation functions are given by

$$
\begin{aligned}
\int d p_{1}^{-} \mathcal{W}^{+i ;+-}\left(P_{1}, p_{1}\right) & =2 P_{1}^{+} p_{1}^{-} p_{1}^{i} \frac{Z^{2} \alpha}{\pi^{2}}\left|\frac{F\left(-p_{1}^{2}\right)}{-p_{1}^{2}}\right|^{2}, \\
\int d p_{1}^{-} \mathcal{W}^{i j ; k+}\left(P_{1}, p_{1}\right) & =0
\end{aligned}
$$

and the twist- 4 correlation function is $\int d p_{1}^{-} \mathcal{W}^{+-;+-}\left(P_{1}, p_{1}\right)=4 P_{1}^{+}\left(p_{1}^{-}\right)^{2} \frac{Z^{2} \alpha}{\pi^{2}}\left|\frac{F\left(-p_{1}^{2}\right)}{-p_{1}^{2}}\right|^{2}$

No higher order (twist-3 and twist-4) correlation functions contribute to the tree level diagram of $\gamma \gamma \rightarrow \bar{l}$.

The total cross section in the TMD factorization formalism reads

$$
\sigma=\sigma_{\text {twist } 2}+\sigma_{\text {twist } n}
$$

where

$$
\sigma_{\text {twist } n}=\frac{Z^{4} \alpha^{2} v}{8 \pi^{4}} \int \frac{d^{3} k_{1}}{(2 \pi)^{3} 2 E_{k 1}} \frac{d^{3} k_{2}}{(2 \pi)^{3} 2 E_{k 2}} \frac{d \omega_{1}}{\omega_{1}^{2}} \frac{d \omega_{2}}{\omega_{2}^{2}} d^{2} p_{1 T} d^{2} p_{2 T}\left|\frac{F\left(-p_{1}^{2}\right)}{-p_{1}^{2}}\right|^{2}\left|\frac{F\left(-p_{2}^{2}\right)}{-p_{2}^{2}}\right|^{2}(2 \pi)^{4} \delta^{4}\left(p_{1}+p_{2}-k_{1}-k_{2}\right) \mathcal{I} \text {, }
$$

with $\mathcal{I}$ given by Eq. (36). It is remarkable that $\sigma_{\text {twist } n}$ is exactly $\delta \sigma$ in Eq. (34). Therefore, since we have already shown that $\sigma_{\text {twist } 2}=\sigma_{0}$ in Eq. (43), the total cross section (46) in the TMD factorization formalism is consistent with the EPA result (25).

Thus we have shown the equivalence of EPA and TMD factorization formalism. We would like to emphasize that our main result (20) includes corrections beyond the EPA or twist-2. Note that, in principle, one also needs to consider the Sudakov factor in the TMD factorization theorem $[80,81,86,87]$. It is still a challenge to add the effective
Sudakov factor into our framework, which we reserve for a future study.

\section{NUMERICAL RESULTS}

In this section, we will present numerical results for the cross section and compare them with STAR measurements at $200 \mathrm{GeV} \mathrm{Au}+\mathrm{Au}$ collisions at RHIC [67,69]. The cross section can be expressed as a multidimension integral over independent variables

$$
\begin{aligned}
\sigma= & \frac{Z^{4} \alpha^{2}}{64 \pi^{4} \gamma^{4} v^{2}} \int d b_{T} d \phi_{b} \frac{d \Delta_{T} d \phi_{\Delta}}{(2 \pi)^{2}} \frac{d p_{1 T} d \phi_{p 1}}{(2 \pi)^{2}} \int d Y_{e e} d P_{e e}^{T} d \phi_{e e} d M_{e e} d \eta_{k 2} d \phi_{k 2} \\
& \times e^{-i \mathbf{b}_{T} \cdot \mathbf{\Delta}_{T}} b_{T} \Delta_{T} p_{1 T} \mathcal{P}^{2}\left(b_{T}\right) \mathcal{J} \frac{F^{*}\left(-p_{1}^{\prime 2}\right)}{-p_{1}^{\prime 2}} \frac{F\left(-p_{1}^{2}\right)}{-p_{1}^{2}} \frac{F^{*}\left(-p_{2}^{\prime 2}\right)}{-p_{2}^{\prime 2}} \frac{F\left(-p_{2}^{2}\right)}{-p_{2}^{2}} \\
& \times \sum_{\text {spin of } l, \bar{l}}\left[u_{1 \mu} u_{2 \nu} L^{\mu \nu}\left(p_{1}, p_{2} ; k_{1}, k_{2}\right)\right]\left[u_{1 \sigma} u_{2 \rho} L^{\sigma \rho *}\left(p_{1}^{\prime}, p_{2}^{\prime} ; k_{1}, k_{2}\right)\right] .
\end{aligned}
$$

The derivation of the above formula as well as the explanation of all variables are given in Appendix D. Here we have introduced a factor $\mathcal{P}^{2}\left(b_{T}\right)$ which will be explained shortly. The high-dimension integral of the cross section (48) is a challenge in the numerical calculation. In this paper we use the ZMCintegral package [99,100] which has been applied to the calculation of high dimensional integrals in heavy ion collisions [101].
For the numerical calculation we choose the nucleus form factor $[73,80,81,102]$ as

$$
F(q)=\frac{4 \pi \rho^{0}}{q^{3} A}\left[\sin \left(q R_{A}\right)-q R_{A} \cos \left(q R_{A}\right)\right] \frac{1}{a^{2} q^{2}+1}
$$

where $a=0.7 \mathrm{fm}, R_{A}=1.2 A^{1 / 3} \mathrm{fm}$ is the nucleus radius with $A$ being the number of nucleons, and $\rho^{0}=3 A /\left(4 \pi R_{A}^{3}\right)$ 
TABLE I. The total cross sections from STAR measurements and theoretical models. The numerical integration errors are labeled as "int."

\begin{tabular}{lc}
\hline \hline Data or models & Total cross sections \\
\hline STAR data [67] & $0.261 \pm 0.004$ (stat.) \pm 0.013 (sys.) \\
& \pm 0.034 (scale) $\mathrm{mb}$ \\
STARLight [73] & $0.22 \mathrm{mb}$ \\
Zha et al.'s gEPA [77] & $0.26 \mathrm{mb}$ \\
Zha et al.'s work [77] & $0.26 \mathrm{mb}$ \\
This work Eq. (20) & $0.252 \pm 0.0016$ (int.) $\mathrm{mb}$ \\
gEPA Eq. (30) & $0.256 \pm 0.0030$ (int.) $\mathrm{mb}$ \\
\hline \hline
\end{tabular}

is the nucleon number density to make $F(q=0)=1$ [102]. One can also choose $R_{A}=1 \cdot 1 A^{1 / 3} \mathrm{fm}$ as in Refs. [80,81].

Another factor that should be taken into account is that two nuclei may undergo mutual Coulomb excitation and emit neutrons [81]. To consider such effects, we need to introduce an extra factor $\mathcal{P}^{2}\left(b_{T}\right)$ in the calculation of the cross section, where $\mathcal{P}\left(b_{T}\right)$ is the probability of emitting a single neutron from an excited nucleus and can be parametrized as [103]

$$
\mathcal{P}\left(b_{T}\right)=\sum_{N_{\gamma}=1}^{\infty} \frac{1}{N_{\gamma} !} w^{N_{\gamma}} e^{-w}=1-\exp (-w),
$$

where $N_{\gamma}$ denotes the number of photons that can be absorbed by a nucleus, and $w$ is defined by

$$
w=5.45 \times 10^{-5} \frac{Z^{3}(A-Z)}{A^{2 / 3} b_{T}^{2}} .
$$

Note that the probability of emitting more than one neutron is highly suppressed, so multiple neutron emission is usually neglected. The formula (50) has been widely used $[72,76,78,103-105]$. Other choices are $\mathcal{P}\left(b_{T}\right) \simeq w[76]$ and $\mathcal{P}\left(b_{T}\right)=w \exp (-w)[76,78,103,104,106]$. In this work, we choose (50) in our numerical calculation.

We follow STAR experiments $[67,69]$ to choose that the transverse momentum of the electron and that of the positron is greater than $200 \mathrm{MeV}$ but the transverse momentum of the electron-positron pair is less than $100 \mathrm{MeV}$.

In Table I, we show the total cross sections from STAR measurements and some theoretical models. The total cross sections computed from both Eq. (20) (under the approximation $G_{T} \approx 1$ ) and gEPA in Eq. (30) are very close to the experimental results.

In Fig. 3, we plot differential cross sections as functions of the invariant mass of the lepton pair $M_{e e}$. The STAR data [67] as well as the results of some models [73,74] (including our model) are presented. Our results using Eq. (20) under the approximation $G_{T} \approx 1$ (the blue-solid line) are consistent with data. But our results give lower values than those from $\mathrm{gEPA}$.

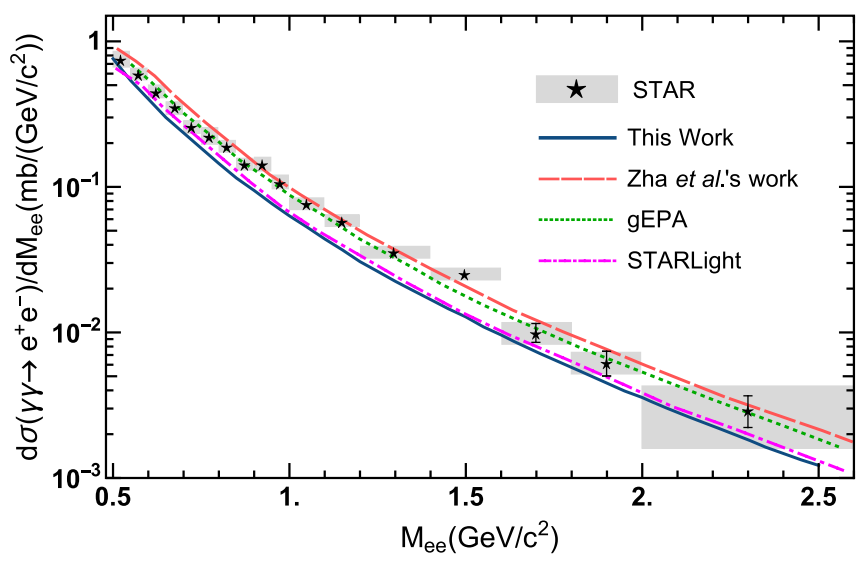

FIG. 3. Differential cross sections as functions of the invariant mass of the lepton pair $M_{e e}$. The blue-solid line represents our results using Eq. (20). The red-dashed, green-dotted, and magenta-dot-dashed lines represent the results from Ref. [74] based on QED, gEPA [74], and STARLight [73], respectively. The points are STAR data [67], while the shaded areas stand for the experimental uncertainty.

The differential cross sections as functions of the lepton pair's transverse momenta are presented in Fig. 4. We compare our results with STAR data [67], the results of Ref. [74], and STARLight [73]. Our results using Eq. (20) are in a good agreement with the data in the low transverse momentum region, while they give a little larger values than the data for transverse momenta larger than $0.02 \mathrm{GeV}$. The difference may come from possible higher order corrections [79,107].

In Fig. 5, we show the average transverse momentum squares of lepton pairs as functions of $M_{e e}$. Our results are

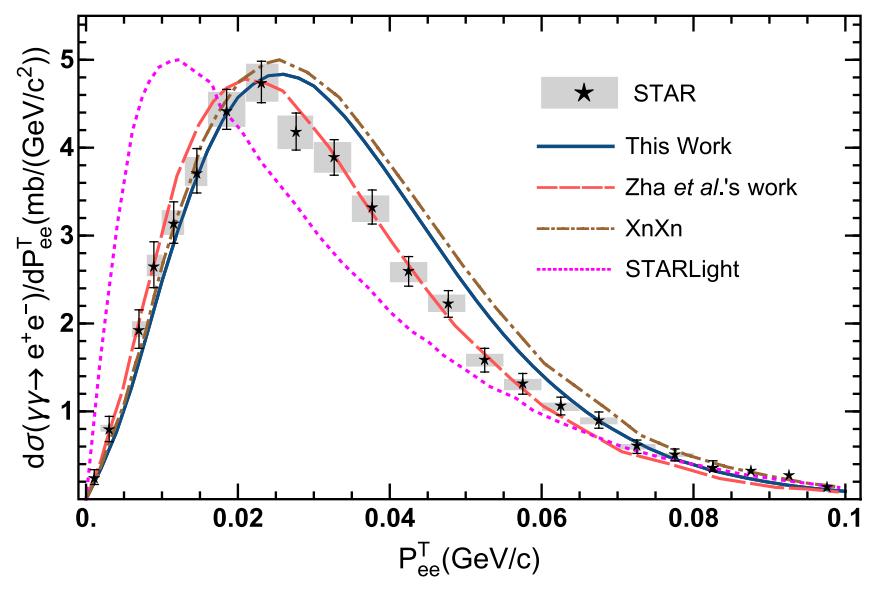

FIG. 4. Differential cross sections as functions of the lepton pair's transverse momenta. The blue-solid line shows our results using Eq. (20). The red-dashed, brown-dot-dashed, and magentadotted lines are the results from Ref. [74] (times a factor of 9.1), XnXn [67], and STARLight [73], respectively. The data points are from STAR measurements [67], while the shaded areas stand for the experimental uncertainty. 


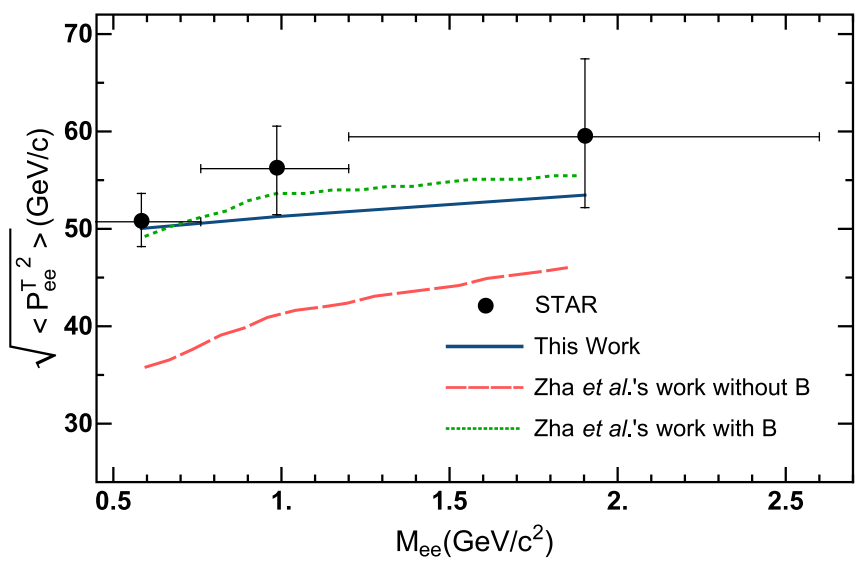

FIG. 5. Average transverse momentum squares of lepton pairs as functions of $M_{e e}$. The blue-solid line shows our results using Eq. (20). The green-dotted lines and red-dashed lines are from Ref. [74] with and without contributions of magnetic fields, respectively. The data points are from STAR measurements [67].

consistent with experimental data, so we do not need to consider extra contributions from magnetic fields at the early stage. According to Refs. $[85,87,90]$, the Sudakov
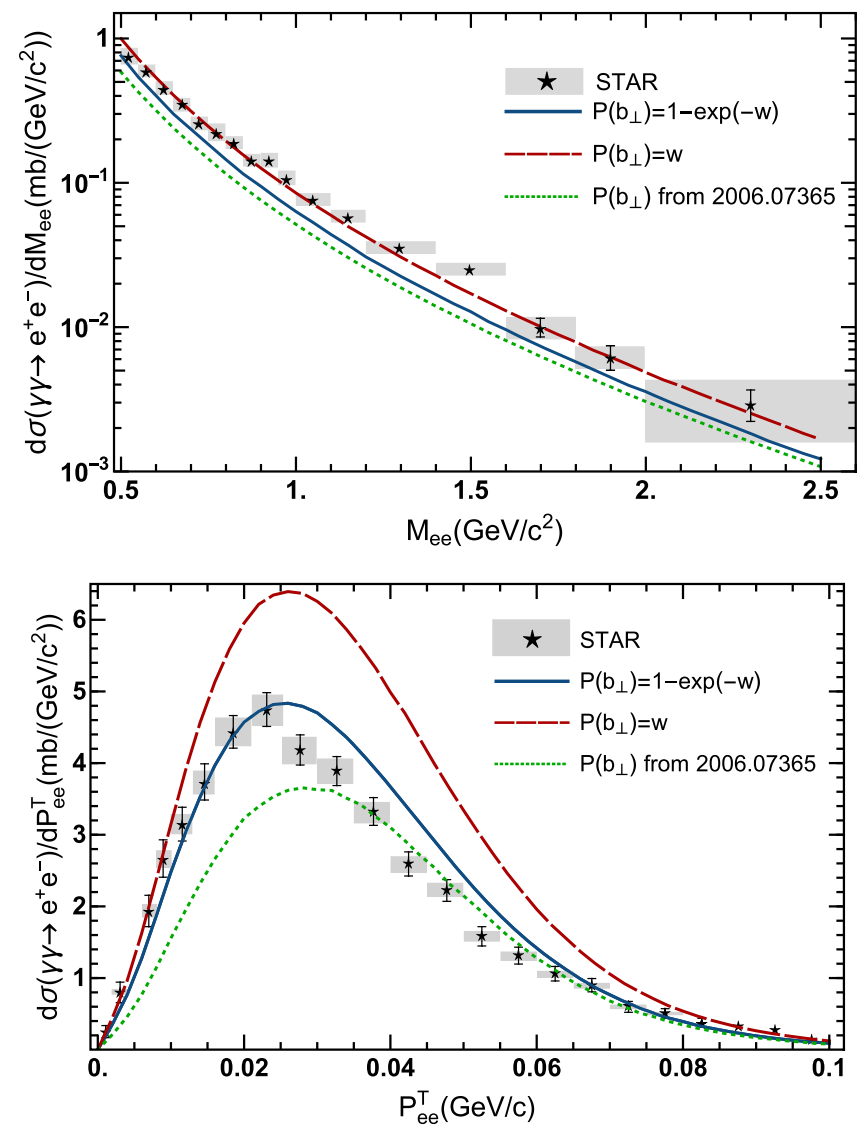

FIG. 6. Differential cross sections by using different $\mathcal{P}\left(b_{T}\right)$. The blue-solid, dark-red-dashed and green-dotted lines are the results of using $\mathcal{P}\left(b_{T}\right)=1-\exp (-w), \mathcal{P}\left(b_{T}\right)=w$, and $\mathcal{P}\left(b_{T}\right)$ of Ref. [78], respectively. form factor may play a role to the broadening of the transverse momentum.

That difference between our results and experimental data may come from the choice of parameters. In Fig. 6, we show the results from different $\mathcal{P}\left(b_{T}\right)$. For the invariant mass spectrum of the lepton pair, the result with $\mathcal{P}\left(b_{T}\right) \simeq w$ [76] matches the data, but the transverse momentum spectrum of the lepton pair is much higher than the data. The result with $\mathcal{P}\left(b_{T}\right)$ of Ref. [78] underestimates both invariant mass and transverse momentum spectra. These results imply that we need to study the parameter dependence systematically, which we leave for a future study.

\section{SUMMARY}

A general form for the cross section of the lepton pair production in ultraperipheral collisions of heavy ions is derived. The wave functions of two colliding nuclei moving in the $\pm z$ direction are assumed to be in the form of wave packets that allow a rigorous description of ultraperipheral collisions. A relative phase factor is introduced into the wave function of one colliding nucleus that is displaced by an impact parameter from the other colliding nucleus, through which the transverse momentum and position are coupled. This leads to photon distributions with dependence on transverse momentum and position.

The results of the generalized equivalent photon approximation can be reproduced at the ultrarelativistic limit in our formalism. The results of the TMD factorization formalism up to the Born approximation can also be reproduced from our general form if light-cone coordinates are used. It can be proved that the results of the generalized equivalent photon approximation are consistent to the twist- 2 results of the TMD factorization formalism in the classical field approximation at the Born level. We have also shown that the general form of the cross section has already included high order corrections such as the ones beyond the equivalent photon approximation or the ones from higher twists in the classical field approximation at the Born level.

The numerical results for the differential cross sections with respect to invariant mass and transverse momentum of the lepton pair are in a good agreement with STAR data. One approximation we have made in our results is that wave packets are assumed to be narrow so that they are similar to plane waves. In the future this approximation will be relaxed so that the effects from broader wave packets can be studied. Other effects such as the Sudakov factor as well as the dependence on various parameters are expected to be investigated in the future.

\section{ACKNOWLEDGMENTS}

We thank Jian Zhou, Zhangbu Xu, Wangmei Zha, and Zebo Tang for helpful discussion. S. P. is supported by National Nature Science Foundation of China (NSFC) under Grant No. 12075235. Q. W. is supported in part 
by NSFC under Grants No. 11890713 (a subgrant of No. 11890710) and No. 11947301, and by the Strategic Priority Research Program of Chinese Academy of Sciences under Grant No. XDB34030102.

\section{APPENDIX A: DERIVATION OF IMPACT- PARAMETER DEPENDENT CROSS SECTION}

In this Appendix, we will derive the cross section that depends on the impact parameter $\mathbf{b}_{T}$. We assume the wave functions of two colliding nuclei moving in the $\pm z$ direction (see Fig. 2) can be written in the form of wave packets following the argument of Ref. [108],

$$
\begin{aligned}
& \left|A_{1}\right\rangle=\int \frac{d^{3} P_{1}}{(2 \pi)^{3} \sqrt{2 E_{P 1}}} \phi\left(P_{1}\right) e^{i b \cdot P_{1}}\left|P_{1}\right\rangle, \\
& \left|A_{2}\right\rangle=\int \frac{d^{3} P_{2}}{(2 \pi)^{3} \sqrt{2 E_{P 2}}} \phi\left(P_{2}\right)\left|P_{2}\right\rangle,
\end{aligned}
$$

where $P_{1}=\left(E_{P 1}, \mathbf{P}_{1}\right)$ and $P_{2}=\left(E_{P 2}, \mathbf{P}_{2}\right)$ with $E_{P 1}=$ $\sqrt{\mathbf{P}_{1}^{2}+M_{1}^{2}}$ and $E_{P 2}=\sqrt{\mathbf{P}_{2}^{2}+M_{2}^{2}}$ are on-shell momenta of two nuclei with masses $M_{1}$ and $M_{2} ; \phi\left(P_{1}\right) \equiv \phi\left(\mathbf{P}_{1}-\right.$ $\left.\mathbf{P}_{A 1}\right)$ and $\phi\left(P_{2}\right) \equiv \phi\left(\mathbf{P}_{2}-\mathbf{P}_{A 2}\right)$ denote the amplitudes of momentum states that are centered at the nuclear momenta $\quad \mathbf{P}_{A 1}=\left(0,0, P_{A 1}^{z}\right)$ and $\quad \mathbf{P}_{A 2}=\left(0,0,-P_{A 1}^{z}\right)$, respectively. They satisfy the normalization condition $(2 \pi)^{-3} \int d^{3} k|\phi(k)|^{2}=1$. Here we have introduced the impact parameter $b^{\mu}=\left(0, \mathbf{b}_{T}, 0\right)$ into the state of $A_{1}$, which is the transverse distance between the centers of two nuclei.

The initial state of nuclei can be written as

$$
\left|A_{1} A_{2}\right\rangle_{\text {in }}=\int \frac{d^{3} P_{1}}{(2 \pi)^{3}} \frac{d^{3} P_{2}}{(2 \pi)^{3}} \frac{\phi\left(P_{1}\right) \phi\left(P_{2}\right) e^{i b_{T} \cdot P_{1}}}{\sqrt{2 E_{P 1}} \sqrt{2 E_{P 2}}}\left|P_{1} P_{2}\right\rangle_{\text {in }},
$$

while the final state is assumed to be in momentum states instead of wave packets

$$
\left|l, \bar{l}, \sum_{f} X_{f}\right\rangle \equiv\left|k_{1}, k_{2}, \sum_{f} K_{f}\right\rangle_{\text {out }},
$$

where the indices "in" and "out" stand for the in and out states at $t \rightarrow \pm \infty$, respectively, and $X_{f}$ with momenta $K_{f}$ denote all other particles that are produced in collisions.

The cross section reads

$$
\begin{aligned}
\sigma= & \int d^{2} \mathbf{b}_{T} \sum_{\{f\}} \int \frac{d^{3} k_{1}}{(2 \pi)^{3} 2 E_{k_{1}}} \frac{d^{3} k_{2}}{(2 \pi)^{3} 2 E_{k_{2}}} \prod_{f} \frac{d^{3} K_{f}}{(2 \pi)^{3} 2 E_{f}} \\
& \times\left.\left.\right|_{\text {out }}\left\langle k_{1}, k_{2}, \sum_{f} K_{f} \mid A_{1} A_{2}\right\rangle_{\text {in }}\right|^{2} .
\end{aligned}
$$

Using Eqs. (A2) and (A12) and completing the integration over $P_{1}^{z z}$ and $P_{2}^{z z}$ in the above formula, we obtain

$$
\begin{aligned}
\sigma= & \int d^{2} \mathbf{b}_{T} \sum_{\{f\}} \int \frac{d^{3} k_{1}}{(2 \pi)^{3} 2 E_{k 1}} \frac{d^{3} k_{2}}{(2 \pi)^{3} 2 E_{k 2}} \prod_{f} \frac{d^{3} K_{f}}{(2 \pi)^{3} 2 E_{K f}} \int \frac{d^{3} P_{1}}{(2 \pi)^{3} \sqrt{2 E_{P 1}}} \frac{d^{3} P_{2}}{(2 \pi)^{3} \sqrt{2 E_{P 2}}} \frac{d^{3} P_{1}^{\prime}}{(2 \pi)^{3} \sqrt{2 E_{P 1^{\prime}}}} \frac{d^{3} P_{2}^{\prime}}{(2 \pi)^{3} \sqrt{2 E_{P 2^{\prime}}}} \\
& \times \phi\left(P_{1}\right) \phi\left(P_{2}\right) \phi^{*}\left(P_{1}^{\prime}\right) \phi^{*}\left(P_{2}^{\prime}\right) e^{-i \mathbf{b}_{T} \cdot \mathbf{\Delta}_{1 T}}(2 \pi)^{4} \delta^{(4)}\left(P_{1}+P_{2}-k_{1}-k_{2}-\sum_{f} K_{f}\right)(2 \pi)^{4} \delta^{(4)}\left(P_{1}+P_{2}-P_{1}^{\prime}-P_{2}^{\prime}\right) \\
& \times \sum_{\text {spin of } l, \bar{l}} \mathcal{M}_{P_{1}+P_{2} \rightarrow k_{1}+k_{2}+\sum_{f} K_{f}} \mathcal{M}_{P_{1}^{\prime}+P_{2}^{\prime} \rightarrow k_{1}+k_{2}+\sum_{f} K_{f}}^{*}
\end{aligned}
$$

where $\boldsymbol{\Delta}_{1 T} \equiv \mathbf{P}_{1 T}^{\prime}-\mathbf{P}_{1 T}$ and $\mathcal{M}$ denotes the invariant amplitude defined through the $T$-matrix element. By rewriting the delta function for transverse momenta as

$$
\delta^{(2)}\left(\mathbf{P}_{1 T}+\mathbf{P}_{2 T}-\mathbf{P}_{1 T}^{\prime}-\mathbf{P}_{2 T}^{\prime}\right)=\int \frac{d^{2} \mathbf{b}_{2 T}}{(2 \pi)^{2}} \exp \left[i \mathbf{b}_{2 T} \cdot\left(\mathbf{P}_{1 T}+\mathbf{P}_{2 T}-\mathbf{P}_{1 T}^{\prime}-\mathbf{P}_{2 T}^{\prime}\right)\right]
$$

and adding an integral

$$
\int d^{2} \mathbf{b}_{1 T} \delta^{(2)}\left(\mathbf{b}_{T}-\mathbf{b}_{1 T}+\mathbf{b}_{2 T}\right)=1
$$

the cross section (A5) can now be put into the form 


$$
\begin{aligned}
\sigma= & \int d^{2} \mathbf{b}_{T} d^{2} \mathbf{b}_{1 T} d^{2} \mathbf{b}_{2 T} \sum_{\{f\}} \int \frac{d^{3} k_{1}}{(2 \pi)^{3} 2 E_{k 1}} \frac{d^{3} k_{2}}{(2 \pi)^{3} 2 E_{k 2}} \prod_{f} \frac{d^{3} K_{f}}{(2 \pi)^{3} 2 E_{K f}} \\
& \times \int \frac{d^{3} P_{1}}{(2 \pi)^{3} \sqrt{2 E_{P 1}}} \frac{d^{3} P_{2}}{(2 \pi)^{3} \sqrt{2 E_{P 2}}} \frac{d^{3} P_{1}^{\prime}}{(2 \pi)^{3} \sqrt{2 E_{P 1^{\prime}}}} \frac{d^{3} P_{2}^{\prime}}{(2 \pi)^{3} \sqrt{2 E_{P 2^{\prime}}}} \phi\left(P_{1}\right) \phi\left(P_{2}\right) \phi^{*}\left(P_{1}^{\prime}\right) \phi^{*}\left(P_{2}^{\prime}\right) \\
& \times e^{-i \mathbf{b}_{1 T} \cdot \Delta_{1 T}} e^{-i \mathbf{b}_{2 T} \cdot \Delta_{2 T}} \delta^{(2)}\left(\mathbf{b}_{T}-\mathbf{b}_{1 T}+\mathbf{b}_{2 T}\right)(2 \pi)^{4} \delta^{(4)}\left(P_{1}+P_{2}-k_{1}-k_{2}-\sum_{f} K_{f}\right)(2 \pi)^{2} \delta\left(P_{1}^{\prime z}+P_{2}^{\prime z}-P_{1}^{z}-P_{2}^{z}\right) \\
& \times \delta\left(E_{P 1^{\prime}}+E_{P 2^{\prime}}-E_{P 1}-E_{P 2}\right) \sum_{\text {spin of } l, \bar{l}} \mathcal{M}_{P_{1}+P_{2} \rightarrow k_{1}+k_{2}+\sum_{f} K_{f}} \mathcal{M}_{P_{1}^{\prime}+P_{2}^{\prime} \rightarrow k_{1}+k_{2}+\sum_{f} K_{f}},
\end{aligned}
$$

where we have used $\boldsymbol{\Delta}_{2 T} \equiv \mathbf{P}_{2 T}^{\prime}-\mathbf{P}_{2 T}$.

Now we look at the momentum amplitudes $\phi\left(P_{1}\right)$ and $\phi\left(P_{2}\right)$ in nuclear wave functions as wave packets. Normally the total cross section does not depend on the form of $\phi\left(P_{1}\right)$ and $\phi\left(P_{2}\right)$ [108]. A natural choice for $\phi\left(P_{1}\right)$ and $\phi\left(P_{2}\right)$ is that they can be factorized as $\phi\left(P_{i}\right) \simeq \phi_{z}\left(P_{i}^{z}\right) \phi_{T}\left(\mathbf{P}_{i T}\right)$ with $i=1,2$, where $\phi_{z}\left(P_{i}^{z}\right)$ are distributions of longitudinal momenta that $P_{1}^{z}$ and $P_{2}^{z}$ are centered at $P_{A 1}^{z}$ and $-P_{A 1}^{z}$, respectively, and $\phi_{T}\left(\mathbf{P}_{i T}\right)$ are distributions of transverse momenta that $\mathbf{P}_{i T}$ are centered at zero. As a simple ansatz we assume the longitudinal part takes the form

$\phi_{z}\left(P_{1}^{z}\right) \phi_{z}\left(P_{2}^{z}\right) \phi_{z}^{*}\left(P_{1}^{\prime z}\right) \phi_{z}^{*}\left(P_{2}^{\prime z}\right) \approx(2 \pi)^{2} \delta\left[\left(P_{1}^{z}+P_{1}^{\prime z}\right) / 2-P_{A 1}^{z}\right] \delta\left[\left(P_{2}^{z}+P_{2}^{\prime z}\right) / 2+P_{A 1}^{z}\right] G\left[\frac{1}{4}\left(P_{1}^{z}-P_{1}^{\prime z}\right)^{2}\right] G\left[\frac{1}{4}\left(P_{2}^{z}-P_{2}^{\prime z}\right)^{2}\right]$,

where $G\left(x^{2}\right)$ is a positive function with $G(0)=1$ and decreases rapidly with growing $x^{2}$, and the factor of $1 / 4$ inside $G$-functions is by convention. So we can carry out the integrals over $P_{1}^{z}$ and $P_{2}^{z}$ to remove two delta functions and to set $P_{1}^{z}=2 P_{A 1}^{z}-P_{1}^{\prime z}$ and $P_{2}^{z}=-2 P_{A 1}^{z}-P_{2}^{\prime z}$ in the integrand. Then we complete the integrals over $P_{1}^{\prime z}$ and $P_{2}^{\prime z}$ to remove two delta functions for longitudinal momenta and energies

$$
\begin{aligned}
\int d P_{1 z}^{\prime} d P_{2 z}^{\prime} \delta\left(P_{1}^{\prime z}+P_{2}^{\prime z}-P_{1}^{z}-P_{2}^{z}\right) \delta\left(E_{P 1^{\prime}}+E_{P 2^{\prime}}-E_{P 1}-E_{P 2}\right) & =\frac{1}{2} \int d P_{1 z}^{\prime} d P_{2 z}^{\prime} \delta\left(P_{1}^{\prime z}+P_{2}^{\prime z}\right) \delta\left(E_{P 1^{\prime}}+E_{P 2^{\prime}}-E_{P 1}-E_{P 2}\right) \\
& =\frac{1}{2\left|P_{1}^{\prime z} / E_{P 1^{\prime}}+P_{1}^{\prime z} / E_{P 2^{\prime}}+P_{1}^{z} / E_{P 1}+P_{1}^{z} / E_{P 2}\right|} \equiv \frac{1}{8 v}
\end{aligned}
$$

where we have used $P_{1}^{z}=2 P_{A 1}^{z}-P_{1}^{\prime z}$ and $P_{2}^{z}=-2 P_{A 1}^{z}-P_{2}^{\prime z}$, and $P_{1}^{\prime z}=-P_{2}^{\prime z}$ are solved as functions of transverse momenta from the energy conservation. Furthermore the condition $P_{1}^{\prime z}=-P_{2}^{\prime z}$ leads to $P_{1}^{z}=-P_{2}^{z}=2 P_{A 1}^{z}-P_{1}^{\prime z}$. With these conditions, two $G$-functions in Eq. (A9) give $G^{2}\left[\left(P_{1}^{\prime z}-P_{A 1}^{z}\right)^{2}\right]$ as a function of transverse momenta.

Completing the integrals over $P_{1}^{z}, P_{2}^{z}, P_{1}^{z}$, and $P_{2}^{\prime z}$ in Eq. (A8) by using (A9) and (A10), we obtain Eq. (2) for the cross section.

Now we deal with the invariant amplitude $\mathcal{M}$ through the matrix element of the operator $\hat{T}$ ( $T$-matrix element) as

$$
{ }_{\text {out }}\left\langle k_{1}, k_{2}, \sum_{f} K_{f} \mid P_{1} P_{2}\right\rangle_{\text {in }}=\left\langle k_{1}, k_{2}, \sum_{f} K_{f}|(1+i \hat{T})| P_{1} P_{2}\right\rangle
$$

with the $T$-matrix element being parametrized as

$$
\left\langle k_{1}, k_{2}, \sum_{f} K_{f}|i \hat{T}| P_{1} P_{2}\right\rangle=(2 \pi)^{4} \delta^{(4)}\left(P_{1}+P_{2}-k_{1}-k_{2}-\sum_{f} K_{f}\right) i \mathcal{M}_{P_{1}+P_{2} \rightarrow k_{1}+k_{2}+\sum_{f} K_{f}}
$$


where $\mathcal{M}$ denotes the invariant amplitude of the process. By definition, the $T$-matrix element can be written in the form

$$
\left\langle k_{1}, k_{2}, \sum_{f} K_{f}|i \hat{T}| P_{1} P_{2}\right\rangle=-e^{2} \int d^{4} x_{1} d^{4} x_{2}\left\langle k_{1} k_{2}\left|\mathcal{T}\left[\bar{\psi}\left(x_{1}\right) \gamma^{\mu} \psi\left(x_{1}\right) \bar{\psi}_{2}\left(x_{2}\right) \gamma^{\nu} \psi_{2}\left(x_{2}\right)\right]\right| 0\right\rangle\left\langle\sum_{f} K_{f}\left|\mathcal{T}\left[A_{\mu}\left(x_{1}\right) A_{\nu}\left(x_{2}\right)\right]\right| P_{1} P_{2}\right\rangle,
$$

where $\mathcal{T}$ denotes the time-ordered operator. The lepton part is evaluated as

$$
\begin{aligned}
\left\langle k_{1} k_{2}\left|\mathcal{T}\left[\bar{\psi}\left(x_{1}\right) \gamma^{\mu} \psi\left(x_{1}\right) \bar{\psi}_{2}\left(x_{2}\right) \gamma^{\nu} \psi_{2}\left(x_{2}\right)\right]\right| 0\right\rangle= & i \int \frac{d^{4} q}{(2 \pi)^{4}} \bar{u}\left(k_{1}\right) \gamma^{\mu} \frac{\gamma \cdot q+m}{q^{2}-m^{2}+i \varepsilon} \gamma^{\nu} v\left(k_{2}\right) e^{i k_{1} \cdot x_{1}+i k_{2} \cdot x_{2}-i q \cdot\left(x_{1}-x_{2}\right)} \\
& +i \int \frac{d^{4} q}{(2 \pi)^{4}} \bar{u}\left(k_{1}\right) \gamma^{\nu} \frac{\gamma \cdot q+m}{q^{2}-m^{2}+i \varepsilon} \gamma^{\mu} v\left(k_{2}\right) e^{i k_{1} \cdot x_{2}+i k_{2} \cdot x_{1}+i q \cdot\left(x_{1}-x_{2}\right)} .
\end{aligned}
$$

We make an approximation that the time-ordered operator in the EM part can be removed so that it can be put into the form

$$
\begin{aligned}
\left\langle\sum_{f} K_{f}\left|\mathcal{T}\left[A_{\mu}\left(x_{1}\right) A_{\nu}\left(x_{2}\right)\right]\right| P_{1} P_{2}\right\rangle & \sim\left\langle\sum_{f} K_{f}\left|A_{\mu}\left(x_{1}\right) A_{\nu}\left(x_{2}\right)\right| P_{1} P_{2}\right\rangle \\
& =\int \frac{d^{4} p_{1}}{(2 \pi)^{4}} \int \frac{d^{4} p_{2}}{(2 \pi)^{4}} \exp \left(-i p_{1} \cdot x_{1}-i p_{2} \cdot x_{2}\right)\left\langle\sum_{f} K_{f}\left|A_{\mu}\left(p_{1}\right) A_{\nu}\left(p_{2}\right)\right| P_{1} P_{2}\right\rangle
\end{aligned}
$$

where we have performed Fourier transformation for the EM fields. Using Eqs. (A14) and (A15) and completing the integrals over $x_{1}$ and $x_{2}$ in Eq. (A13), we obtain

$$
\begin{aligned}
\left\langle k_{1}, k_{2}, \sum_{f} K_{f}|i \hat{T}| P_{1} P_{2}\right\rangle= & (2 \pi)^{4} \delta^{(4)}\left(P_{1}+P_{2}-k_{1}-k_{2}-\sum_{f} K_{f}\right) i \mathcal{M}_{P_{1}+P_{2} \rightarrow k_{1}+k_{2}+\sum_{f} K_{f},} \\
\mathcal{M}_{P_{1}+P_{2} \rightarrow k_{1}+k_{2}+\sum_{f} K_{f}}= & -e^{2} \int \frac{d^{4} p_{1}}{(2 \pi)^{4}} \int \frac{d^{4} p_{2}}{(2 \pi)^{4}}\left\langle\sum_{f} K_{f}\left|A_{\mu}\left(p_{1}\right) A_{\nu}\left(p_{2}\right)\right| P_{1} P_{2}\right\rangle \\
& \times\left[\bar{u}\left(k_{1}\right) \gamma^{\mu} \frac{\gamma \cdot\left(k_{1}-p_{1}\right)+m}{\left(k_{1}-p_{1}\right)^{2}-m^{2}+i \varepsilon} \gamma^{\nu} v\left(k_{2}\right)+\bar{u}\left(k_{1}\right) \gamma^{\nu} \frac{\gamma \cdot\left(p_{1}-k_{2}\right)+m}{\left(p_{1}-k_{2}\right)^{2}-m^{2}+i \varepsilon} \gamma^{\mu} v\left(k_{2}\right)\right] .
\end{aligned}
$$

Following the same procedure, we obtain

$$
\begin{aligned}
\left\langle P_{1}^{\prime} P_{2}^{\prime}\left|\left(-i \hat{T}^{\dagger}\right)\right| k_{1}, k_{2}, \sum_{f} K_{f}\right\rangle= & (2 \pi)^{4} \delta^{(4)}\left(P_{1}^{\prime}+P_{2}^{\prime}-k_{1}-k_{2}-\sum_{f} K_{f}\right)(-i) \mathcal{M}_{P_{1}^{\prime}+P_{2}^{\prime} \rightarrow k_{1}+k_{2}+\sum_{f} K_{f}}^{*} \\
\mathcal{M}_{P_{1}^{\prime}+P_{2}^{\prime} \rightarrow k_{1}+k_{2}+\sum_{f} K_{f}}^{*}= & -e^{2} \int \frac{d^{4} p_{1}^{\prime}}{(2 \pi)^{4}} \int \frac{d^{4} p_{2}^{\prime}}{(2 \pi)^{4}}\left\langle P_{1}^{\prime} P_{2}^{\prime}\left|A_{\nu}^{\dagger}\left(p_{2}^{\prime}\right) A_{\mu}^{\dagger}\left(p_{1}^{\prime}\right)\right| \sum_{f} K_{f}\right\rangle \\
& \times\left[\bar{v}\left(k_{2}\right) \gamma^{\nu} \frac{\gamma \cdot\left(k_{1}-p_{1}^{\prime}\right)+m}{\left(k_{1}-p_{1}^{\prime}\right)^{2}-m^{2}+i \varepsilon} \gamma^{\mu} u\left(k_{1}\right)+\bar{v}\left(k_{2}\right) \gamma^{\mu} \frac{\gamma \cdot\left(p_{1}^{\prime}-k_{2}\right)+m}{\left(p_{1}^{\prime}-k_{2}\right)^{2}-m^{2}+i \varepsilon} \gamma^{\nu} u\left(k_{1}\right)\right] .
\end{aligned}
$$

Note that in Eqs. (A16) and (A17) we have suppressed the spin indices of lepton spinors. 
Using Eqs. (A16) and (A17) in Eq. (2), the cross section is put into the form

$$
\begin{aligned}
\sigma= & \frac{1}{8(2 \pi)^{8}} \int d^{2} \mathbf{b}_{T} d^{2} \mathbf{b}_{1 T} d^{2} \mathbf{b}_{2 T} \int \frac{d^{3} k_{1}}{(2 \pi)^{3} 2 E_{k 1}} \frac{d^{3} k_{2}}{(2 \pi)^{3} 2 E_{k 2}} \int d^{2} \mathbf{P}_{1 T} d^{2} \mathbf{P}_{2 T} d^{2} \mathbf{P}_{1 T}^{\prime} d^{2} \mathbf{P}_{2 T}^{\prime} \frac{1}{v \sqrt{E_{P 1} E_{P 2} E_{P 1^{\prime}} E_{P 2^{\prime}}}} \\
& \times \phi_{T}\left(\mathbf{P}_{1 T}\right) \phi_{T}\left(\mathbf{P}_{2 T}\right) \phi_{T}^{*}\left(\mathbf{P}_{1 T}^{\prime}\right) \phi_{T}^{*}\left(\mathbf{P}_{2 T}^{\prime}\right) G^{2}\left[\left(P_{1}^{\prime z}-P_{A 1}^{z}\right)^{2}\right] e^{-i \mathbf{b}_{1 T} \cdot \mathbf{\Delta}_{1 T}} e^{-i \mathbf{b}_{2 T} \cdot \mathbf{\Delta}_{2 T}} \delta^{(2)}\left(\mathbf{b}_{T}-\mathbf{b}_{1 T}+\mathbf{b}_{2 T}\right) \\
& \times \int \frac{d^{4} p_{1}}{(2 \pi)^{4}} \frac{d^{4} p_{2}}{(2 \pi)^{4}} \frac{d^{4} p_{1}^{\prime}}{(2 \pi)^{4}} \frac{d^{4} p_{2}^{\prime}}{(2 \pi)^{4}}\left\langle P_{1}^{\prime}\left|A_{\sigma}^{\dagger}\left(p_{1}^{\prime}\right) A_{\mu}\left(p_{1}\right)\right| P_{1}\right\rangle\left\langle P_{2}^{\prime}\left|A_{\rho}^{\dagger}\left(p_{2}^{\prime}\right) A_{\nu}\left(p_{2}\right)\right| P_{2}\right\rangle \\
& \times L^{\mu \nu ; \sigma \rho}\left(p_{1}, p_{2} ; p_{1}^{\prime}, p_{2}^{\prime} ; k_{1}, k_{2}\right)(2 \pi)^{4} \delta^{(4)}\left(p_{1}+p_{2}-k_{1}-k_{2}\right),
\end{aligned}
$$

where we have used the identity

$$
\sum_{\{f\}} \int \prod_{f} \frac{d^{3} K_{f}}{(2 \pi)^{3} 2 E_{K f}}\left|\sum_{f} K_{f}\right\rangle\left\langle\sum_{f} K_{f}\right|=1
$$

and the relation

$$
\sum_{f} K_{f}=P_{1}+P_{2}-p_{1}-p_{2}=P_{1}^{\prime}+P_{2}^{\prime}-p_{1}^{\prime}-p_{2}^{\prime}
$$

We have also used the lepton part as

$$
\begin{aligned}
L^{\mu \nu ; \sigma \rho}\left(p_{1}, p_{2} ; p_{1}^{\prime}, p_{2}^{\prime} ; k_{1}, k_{2}\right)= & e^{4} \sum_{\text {spin of } l, \bar{l}} L^{\mu \nu}\left(p_{1}, p_{2} ; k_{1}, k_{2}\right) L^{\sigma \rho *}\left(p_{1}^{\prime}, p_{2}^{\prime} ; k_{1}, k_{2}\right) \\
= & e^{4} \operatorname{Tr}\left\{\left(\gamma \cdot k_{1}+m\right)\left[\gamma^{\mu} \frac{\gamma \cdot\left(k_{1}-p_{1}\right)+m}{\left(k_{1}-p_{1}\right)^{2}-m^{2}+i \varepsilon} \gamma^{\nu}+\gamma^{\nu} \frac{\gamma \cdot\left(p_{1}-k_{2}\right)+m}{\left(p_{1}-k_{2}\right)^{2}-m^{2}+i \varepsilon} \gamma^{\mu}\right]\right. \\
& \left.\times\left(\gamma \cdot k_{2}-m\right)\left[\gamma^{\rho} \frac{\gamma \cdot\left(k_{1}-p_{1}^{\prime}\right)+m}{\left(k_{1}-p_{1}^{\prime}\right)^{2}-m^{2}+i \varepsilon} \gamma^{\sigma}+\gamma^{\sigma} \frac{\gamma \cdot\left(p_{1}^{\prime}-k_{2}\right)+m}{\left(p_{1}^{\prime}-k_{2}\right)^{2}-m^{2}+i \varepsilon} \gamma^{\rho}\right]\right\}
\end{aligned}
$$

where $L^{\mu \nu}$ and $L^{\sigma \rho *}$ are defined by

$$
\begin{aligned}
L^{\mu \nu}\left(p_{1}, p_{2} ; k_{1}, k_{2}\right) & =\bar{u}\left(k_{1}\right)\left[\gamma^{\mu} \frac{\gamma \cdot\left(k_{1}-p_{1}\right)+m}{\left(k_{1}-p_{1}\right)^{2}-m^{2}+i \varepsilon} \gamma^{\nu}+\gamma^{\nu} \frac{\gamma \cdot\left(p_{1}-k_{2}\right)+m}{\left(p_{1}-k_{2}\right)^{2}-m^{2}+i \varepsilon} \gamma^{\mu}\right] v\left(k_{2}\right), \\
L^{\sigma \rho *}\left(p_{1}^{\prime}, p_{2}^{\prime} ; k_{1}, k_{2}\right) & =\bar{v}\left(k_{2}\right)\left[\gamma^{\rho} \frac{\gamma \cdot\left(k_{1}-p_{1}^{\prime}\right)+m}{\left(k_{1}-p_{1}^{\prime}\right)^{2}-m^{2}+i \varepsilon} \gamma^{\sigma}+\gamma^{\sigma} \frac{\gamma \cdot\left(p_{1}^{\prime}-k_{2}\right)+m}{\left(p_{1}^{\prime}-k_{2}\right)^{2}-m^{2}+i \varepsilon} \gamma^{\rho}\right] u\left(k_{1}\right) .
\end{aligned}
$$

A useful property of $L^{\mu \nu}\left(p_{1}, p_{2}, k_{1}, k_{2}\right)$ is the following identity [71]:

$$
p_{1 \mu} L^{\mu \nu}=p_{2 \nu} L^{\mu \nu}=0
$$

In (A18) we have also reduced the photon matrix element as

$$
\begin{aligned}
\left\langle P_{1}^{\prime}\right. & \left.P_{2}^{\prime}\left|A_{\rho}^{\dagger}\left(p_{2}^{\prime}\right) A_{\sigma}^{\dagger}\left(p_{1}^{\prime}\right) A_{\mu}\left(p_{1}\right) A_{\nu}\left(p_{2}\right)\right| P_{1} P_{2}\right\rangle \\
\sim & \frac{1}{4}\left\langle P_{1}^{\prime}\left|A_{\sigma}^{\dagger}\left(p_{1}^{\prime}\right) A_{\mu}\left(p_{1}\right)\right| P_{1}\right\rangle\left\langle P_{2}^{\prime}\left|A_{\rho}^{\dagger}\left(p_{2}^{\prime}\right) A_{\nu}\left(p_{2}\right)\right| P_{2}\right\rangle+\frac{1}{4}\left\langle P_{1}^{\prime}\left|A_{\rho}^{\dagger}\left(p_{2}^{\prime}\right) A_{\mu}\left(p_{1}\right)\right| P_{1}\right\rangle\left\langle P_{2}^{\prime}\left|A_{\sigma}^{\dagger}\left(p_{1}^{\prime}\right) A_{\nu}\left(p_{2}\right)\right| P_{2}\right\rangle \\
\quad & +\frac{1}{4}\left\langle P_{1}^{\prime}\left|A_{\sigma}^{\dagger}\left(p_{1}^{\prime}\right) A_{\nu}\left(p_{2}\right)\right| P_{1}\right\rangle\left\langle P_{2}^{\prime}\left|A_{\rho}^{\dagger}\left(p_{2}^{\prime}\right) A_{\mu}\left(p_{1}\right)\right| P_{2}\right\rangle+\frac{1}{4}\left\langle P_{1}^{\prime}\left|A_{\rho}^{\dagger}\left(p_{2}^{\prime}\right) A_{\nu}\left(p_{2}\right)\right| P_{1}\right\rangle\left\langle P_{2}^{\prime}\left|A_{\sigma}^{\dagger}\left(p_{1}^{\prime}\right) A_{\mu}\left(p_{1}\right)\right| P_{2}\right\rangle+\cdots \\
\sim & \left\langle P_{1}^{\prime}\left|A_{\sigma}^{\dagger}\left(p_{1}^{\prime}\right) A_{\mu}\left(p_{1}\right)\right| P_{1}\right\rangle\left\langle P_{2}^{\prime}\left|A_{\rho}^{\dagger}\left(p_{2}^{\prime}\right) A_{\nu}\left(p_{2}\right)\right| P_{2}\right\rangle .
\end{aligned}
$$

This is because each term in the second approximate equality can be proved to be identical after change of variables, giving a symmetry factor of 4 . So one can just take the first term and multiply it by 4 to obtain the photon matrix element. We can rewrite the integrals over $p_{1}, p_{2}, p_{1}^{\prime}$, and $p_{2}^{\prime}$ in (A18) as 


$$
\begin{aligned}
I= & \int \frac{d^{4} p_{1}}{(2 \pi)^{4}} \frac{d^{4} p_{2}}{(2 \pi)^{4}} \frac{d^{4} p_{1}^{\prime}}{(2 \pi)^{4}} \frac{d^{4} p_{2}^{\prime}}{(2 \pi)^{4}} L^{\mu \nu ; \sigma \rho}\left(p_{1}, p_{2} ; p_{1}^{\prime}, p_{2}^{\prime} ; k_{1}, k_{2}\right)\left\langle P_{1}^{\prime}\left|A_{\sigma}^{\dagger}\left(p_{1}^{\prime}\right) A_{\mu}\left(p_{1}\right)\right| P_{1}\right\rangle\left\langle P_{2}^{\prime}\left|A_{\rho}^{\dagger}\left(p_{2}^{\prime}\right) A_{\nu}\left(p_{2}\right)\right| P_{2}\right\rangle \\
= & \int \frac{d^{4} p_{1}}{(2 \pi)^{4}} \frac{d^{4} p_{2}}{(2 \pi)^{4}} \int d^{4} y_{1} d^{4} y_{2} \exp \left(i p_{1} \cdot y_{1}+i p_{2} \cdot y_{2}\right) L^{\mu \nu ; \sigma \rho}\left(p_{1}, p_{2} ; p_{1}-P_{1}+P_{1}^{\prime}, p_{2}-P_{2}+P_{2}^{\prime} ; k_{1}, k_{2}\right) \\
& \times\left\langle P_{1}^{\prime}\left|A_{\sigma}^{\dagger}(0) A_{\mu}\left(y_{1}\right)\right| P_{1}\right\rangle\left\langle P_{2}^{\prime}\left|A_{\rho}^{\dagger}(0) A_{\nu}\left(y_{2}\right)\right| P_{2}\right\rangle .
\end{aligned}
$$

In deriving the above formula, we have converted all photon fields in momentum space to coordinate space as

$$
\begin{aligned}
\left\langle P_{1}^{\prime}\left|A_{\sigma}^{\dagger}\left(p_{1}^{\prime}\right) A_{\mu}\left(p_{1}\right)\right| P_{1}\right\rangle & \rightarrow\left\langle P_{1}^{\prime}\left|A_{\sigma}^{\dagger}\left(x_{1}^{\prime}\right) A_{\mu}\left(x_{1}\right)\right| P_{1}\right\rangle, \\
\left\langle P_{2}^{\prime}\left|A_{\rho}^{\dagger}\left(p_{2}^{\prime}\right) A_{\nu}\left(p_{2}\right)\right| P_{2}\right\rangle & \rightarrow\left\langle P_{2}^{\prime}\left|A_{\rho}^{\dagger}\left(x_{2}^{\prime}\right) A_{\nu}\left(x_{2}\right)\right| P_{2}\right\rangle,
\end{aligned}
$$

and changed all coordinate variables to $X_{i}=\left(x_{i}+x_{i}^{\prime}\right) / 2$ and $y_{i}=x_{i}-x_{i}^{\prime}$ for $i=1,2$; then we carried out the integration over $X_{i}$ after shifting $x_{1}^{\prime}$ and $x_{2}^{\prime}$ for the photon fields in $\left\langle P_{1}^{\prime}\left|A_{\sigma}^{\dagger}\left(x_{1}^{\prime}\right) A_{\mu}\left(x_{1}\right)\right| P_{1}\right\rangle$ and $\left\langle P_{2}^{\prime}\left|A_{\rho}^{\dagger}\left(x_{2}^{\prime}\right) A_{\nu}\left(x_{2}\right)\right| P_{2}\right\rangle$, respectively, by making use of the formula $A^{\mu}(x+y)=e^{i \hat{p} \cdot x} A^{\mu}(y) e^{-i \hat{p} \cdot x}$.

Inserting (A25) into Eq. (A18), the cross section is rewritten as

$$
\begin{aligned}
\sigma= & \frac{1}{8(2 \pi)^{8}} \int d^{2} \mathbf{b}_{T} d^{2} \mathbf{b}_{1 T} d^{2} \mathbf{b}_{2 T} \int \frac{d^{3} k_{1}}{(2 \pi)^{3} 2 E_{k 1}} \frac{d^{3} k_{2}}{(2 \pi)^{3} 2 E_{k 2}} \int d^{2} \mathbf{P}_{1 T} d^{2} \mathbf{P}_{2 T} d^{2} \mathbf{P}_{1 T}^{\prime} d^{2} \mathbf{P}_{2 T}^{\prime} \frac{1}{v \sqrt{E_{P 1} E_{P 2} E_{P 1^{\prime}} E_{P 2^{\prime}}}} \\
& \times \phi_{T}\left(\mathbf{P}_{1 T}\right) \phi_{T}\left(\mathbf{P}_{2 T}\right) \phi_{T}^{*}\left(\mathbf{P}_{1 T}^{\prime}\right) \phi_{T}^{*}\left(\mathbf{P}_{2 T}^{\prime}\right) G^{2}\left[\left(P_{1}^{\prime z}-P_{A 1}^{z}\right)^{2}\right] e^{-i \mathbf{b}_{1 T} \cdot \mathbf{\Delta}_{1 T}} e^{-i \mathbf{b}_{2 T} \cdot \mathbf{\Delta}_{2 T}} \delta^{(2)}\left(\mathbf{b}_{T}-\mathbf{b}_{1 T}+\mathbf{b}_{2 T}\right) \\
& \times \int \frac{d^{4} p_{1}}{(2 \pi)^{4}} \frac{d^{4} p_{2}}{(2 \pi)^{4}} \int d^{4} y_{1} d^{4} y_{2} \exp \left(i p_{1} \cdot y_{1}+i p_{2} \cdot y_{2}\right)\left\langle P_{1}^{\prime}\left|A_{\sigma}^{\dagger}(0) A_{\mu}\left(y_{1}\right)\right| P_{1}\right\rangle\left\langle P_{2}^{\prime}\left|A_{\rho}^{\dagger}(0) A_{\nu}\left(y_{2}\right)\right| P_{2}\right\rangle \\
& \times L^{\mu \nu ; \sigma \rho}\left(p_{1}, p_{2} ; p_{1}-P_{1}+P_{1}^{\prime}, p_{2}-P_{2}+P_{2}^{\prime} ; k_{1}, k_{2}\right)(2 \pi)^{4} \delta^{(4)}\left(p_{1}+p_{2}-k_{1}-k_{2}\right) .
\end{aligned}
$$

Equations (A18) and (A27) are our starting points.

\section{APPENDIX B: SOME USEFUL FORMULA IN LIGHT-CONE COORDINATES}

A four-vector or a four-tensor can be written in lightcone variables. For example, the coordinate is written as $x^{\mu}=\left(x^{+}, x^{-}, \mathbf{x}_{T}\right)$ with $x^{ \pm}=\left(x^{0} \pm x^{3}\right) / \sqrt{2}$ and $\mathbf{x}_{T}=$ $\left(x^{1}, x^{2}\right)$. The Minkowski metric tensor becomes

$$
g_{\mu \nu}=\left(\begin{array}{cccc}
0 & 1 & 0 & 0 \\
1 & 0 & 0 & 0 \\
0 & 0 & -1 & 0 \\
0 & 0 & 0 & -1
\end{array}\right)
$$

with $\mu=+,-, 1,2$. So we have $x_{\mu}=g_{\mu \nu} x^{\nu}=$ $\left(x^{-}, x^{+},-\mathbf{x}_{T}\right)$. It is convenient to decompose an arbitrary four-vector $a^{\mu}$ by lightlike Sudakov vectors $n_{+}^{\mu}$ and $n_{-}^{\mu}$, satisfying $n_{+}^{2}=n_{-}^{2}=0$ and $n_{+} \cdot n_{-}=1$,

$$
a^{\mu}=a^{+} n_{+}^{\mu}+a^{-} n_{-}^{\mu}+a_{T}^{\mu},
$$

where $a^{ \pm}=\left(a^{0} \pm a^{3}\right) / \sqrt{2}$ and $a_{T}^{\mu}=g_{T}^{\mu \nu} a_{\nu}$ with the spacelike transverse projector

$$
g_{T}^{\mu \nu}=g^{\mu \nu}-n_{+}^{\mu} n_{-}^{\nu}-n_{+}^{\nu} n_{-}^{\mu} .
$$

The inner product of two vectors is $a \cdot b=$ $a^{-} b^{+}+a^{+} b^{-}-\boldsymbol{a}_{T} \cdot \boldsymbol{b}_{T}$. The explicit forms of lightlike Sudakov vectors are

$$
\begin{aligned}
& n_{+}^{\mu}=(1,0,0,0)_{\text {light cone }}=\frac{1}{\sqrt{2}}(1,0,0,1)_{\text {normal coordinate }}, \\
& n_{-}^{\mu}=(0,1,0,0)_{\text {light cone }}=\frac{1}{\sqrt{2}}(1,0,0,-1)_{\text {normal coordinate }} .
\end{aligned}
$$

The four-momenta of colliding nuclei can be written as

$$
P_{A 1, A 2}^{\mu}=M_{1,2} u_{1,2}^{\mu}
$$

where $M_{1,2}$ and $u_{1,2}^{\mu}$ are masses and four-velocities of two nuclei, respectively, and $u_{1,2}^{\mu}$ are given by

$$
\begin{aligned}
& u_{1}^{\mu}=\gamma_{1}\left(1,0,0, v_{1}\right), \\
& u_{2}^{\mu}=\gamma_{2}\left(1,0,0,-v_{2}\right),
\end{aligned}
$$


with $\gamma_{1,2}=1 / \sqrt{1-v_{1,2}^{2}}$ being the Lorentz factor. Then the four-momenta of two nuclei can be written in the form

$$
\begin{aligned}
& P_{A 1}^{\mu}=P_{A 1}^{+} n_{+}^{\mu}+\frac{M_{1}^{2}}{2 P_{A 1}^{+}} n_{-}^{\mu}, \\
& P_{A 2}^{\mu}=\frac{M_{2}^{2}}{2 P_{A 2}^{-}} n_{+}^{\mu}+P_{A 2}^{-} n_{-}^{\mu},
\end{aligned}
$$

where light-cone variables are

$P_{A 1}^{+}=\frac{M_{1} \gamma_{1}}{\sqrt{2}}\left(1+v_{1}\right), \quad P_{A 2}^{-}=\frac{M_{2} \gamma_{2}}{\sqrt{2}}\left(1+v_{2}\right)$.

For high energy nuclei with $v_{1,2} \sim 1$, we have $P_{A 1}^{+} \gg M_{1}$ and $P_{A 2}^{-} \gg M_{2}$.

From the classical photon field (11), we have $p_{i} \cdot u_{i}=$ $p_{i}^{\prime} \cdot u_{i}=0$ for $i=1,2$. Then $p_{1}$ and $p_{2}$ can be decomposed as

$$
\begin{aligned}
& p_{1}^{\mu}=p_{1}^{+} n_{+}^{\mu}+p_{1}^{-} n_{-}^{\mu}+p_{1 T}^{\mu}, \\
& p_{2}^{\mu}=p_{2}^{+} n_{+}^{\mu}+p_{2}^{-} n_{-}^{\mu}+p_{2 T}^{\mu},
\end{aligned}
$$

where

$p_{1}^{ \pm}=\frac{\omega_{1}}{\sqrt{2}}\left(1 \pm \frac{1}{v_{1}}\right), \quad p_{2}^{ \pm}=\frac{\omega_{2}}{\sqrt{2}}\left(1 \mp \frac{1}{v_{2}}\right)$

At high energies, we have $v_{1,2} \rightarrow 1$, so it is easy to verify

$\frac{p_{1}^{+}}{\omega_{1}}, \quad \frac{p_{2}^{-}}{\omega_{2}} \sim \mathcal{O}(1), \quad \frac{p_{1}^{-}}{\omega_{1}}, \quad \frac{p_{2}^{+}}{\omega_{2}} \sim \mathcal{O}\left(\gamma^{-2}\right)$.

On the other hand, in the rest frame of a nucleus, it is assumed

$$
p_{i}^{2} \sim \mathcal{O}\left(x_{i}^{2} M_{i}^{2}\right)
$$

and therefore, we have

$$
\frac{\mathbf{p}_{i T}}{\omega_{i}} \sim \frac{x_{i} M_{i}}{\omega_{i}} \sim \mathcal{O}\left(\gamma^{-1}\right),
$$

which means photons are almost on shell.

We assume a collision of two identical nuclei, so we have $v_{1}=v_{2}$. To simplify $u_{1 \mu} u_{2 \nu} L^{\mu \nu}$ in Eq. (17), we use Eq. (A23) and obtain

$$
\begin{aligned}
& L^{0 \nu}\left(p_{1}, p_{2}, k_{1}, k_{2}\right)=\frac{p_{1}^{i} L^{i \nu}+p_{1}^{z} L^{z \nu}}{\omega_{1}}, \\
& L^{\mu 0}\left(p_{1}, p_{2}, k_{1}, k_{2}\right)=\frac{p_{2}^{i} L^{\mu i}+p_{2}^{z} L^{\mu z}}{\omega_{2}},
\end{aligned}
$$

where $i=x, y$ denotes two transverse directions. From Eq. (B10) or $p_{1,2}^{z} / \omega_{1,2}= \pm 1 / v$ (the upper/lower signs correspond to nuclei 1 and 2 , respectively), $u_{1 \mu} u_{2 \nu} L^{\mu \nu}$ can be rewritten as

$u_{1 \mu} u_{2 \nu} L^{\mu \nu}=\gamma^{2} \frac{p_{1}^{i}}{\omega_{1}} \frac{p_{2}^{j}}{\omega_{2}} L^{i j}-\frac{1}{v}\left(\frac{p_{1}^{i}}{\omega_{1}} L^{i z}-\frac{p_{2}^{i}}{\omega_{2}} L^{z i}\right)-\frac{1}{\gamma^{2} v^{2}} L^{z z}$,

where $i, j=x, y$ denote two transverse directions. The result of (B15) is consistent with Ref. [71]. We can also use the light-cone coordinate and obtain

$$
\begin{aligned}
L^{-\nu}\left(p_{1}, p_{2}, k_{1}, k_{2}\right) & =-\frac{p_{1}^{-} L^{+\nu}-p_{1}^{i} L^{i \nu}}{p_{1}^{+}}, \\
L^{\mu+}\left(p_{1}, p_{2}, k_{1}, k_{2}\right) & =-\frac{p_{2}^{+} L^{\mu-}-p_{2}^{j} L^{\mu j}}{p_{2}^{-}} .
\end{aligned}
$$

So we evaluate $u_{1 \mu} u_{2 \nu} L^{\mu \nu}$ as

$$
\begin{aligned}
u_{1 \mu} u_{2 \nu} L^{\mu \nu}= & \gamma^{2} v^{2} \frac{p_{1}^{i}}{\omega_{1}} \frac{p_{2}^{j}}{\omega_{2}} L^{i j} \\
& -2 \gamma^{2} v^{2}\left(\frac{p_{1}^{i}}{\omega_{1}} \frac{p_{2}^{+}}{\omega_{2}} L^{i-}+\frac{p_{1}^{-}}{\omega_{1}} \frac{p_{2}^{j}}{\omega_{2}} L^{+j}\right) \\
& +4 \gamma^{2} v^{2} \frac{p_{1}^{-}}{\omega_{1}} \frac{p_{2}^{+}}{\omega_{2}} L^{+-} .
\end{aligned}
$$

It seems that there is an extra factor $v^{2}$ in the first term of Eq. (B17) in comparison with Eq. (B15), and it is straightforward to prove that Eq. (B17) and (B15) are equivalent. According to Eqs. (B11) and (B13), at the relativistic limit $v \rightarrow 1$ or $\gamma \rightarrow \infty$, the first, second, and third terms of (B17) are $O(1), O\left(\gamma^{-1}\right)$ and $O\left(\gamma^{-2}\right)$, respectively. Therefore the leading order contribution comes from the first term.

\section{APPENDIX C: TMD CORRELATION FUNCTIONS IN CLASSICAL FIELD APPROXIMATION}

The gauge invariant correlation function for an unpolarized nucleus is defined as

$\mathcal{W}^{\mu \nu ; \rho \sigma}(P, p)=\int \frac{d^{4} \xi}{(2 \pi)^{4}} e^{i p \cdot \xi}\left\langle P\left|F^{\mu \nu}(0) F^{\rho \sigma}(\xi)\right| P\right\rangle$,

where $F^{\mu \nu}=\partial^{\mu} A^{\nu}-\partial^{\nu} A^{\mu}$ is the field strength tensor of the EM field, and $P=\left(E_{P}, 0,0, P^{z}\right)$ is the four-momentum of 
a nucleus moving in the $+z$ direction. The requirement that $\mathcal{W}^{\mu \nu ; \rho \sigma}(P, p)$ must be Hermitian and parity-even leads to the following parametrization:

$$
\begin{aligned}
\mathcal{W}^{\mu \nu ; \rho \sigma}(P, p)= & X_{1} \epsilon^{\mu \nu \alpha \beta} \epsilon_{\alpha \beta}^{\rho \sigma}+\frac{X_{2}}{M^{2}} P^{[\mu} g^{\nu][\rho} P^{\sigma]}+\frac{X_{3}}{M^{2}} p^{[\mu} g^{\nu][\rho} p^{\sigma]}+\frac{X_{4}+i X_{5}}{M^{2}} P^{[\mu} g^{\nu][\rho} p^{\sigma]}+\frac{X_{4}-i X_{5}}{M^{2}} p^{[\mu} g^{\nu][\rho} P^{\sigma]} \\
& +\frac{X_{6}}{M^{4}} P^{[\mu} p^{\nu]} P^{[\rho} p^{\sigma]},
\end{aligned}
$$

where $A^{[\mu} B^{\nu]}=A^{\mu} B^{\nu}-B^{\mu} A^{\nu}$ and all coefficients $X_{i}$ are real functions of $P$ and $p$. We work in a light-cone coordinate system and focus on $\mathcal{W}^{+\mu ;+\nu}(P, p)$ which is related to $S_{\sigma \mu}(P, p)$ in (9) in the light-cone gauge

$$
\begin{aligned}
\mathcal{W}^{+\mu ;+\nu}(P, p)= & -g_{T}^{\mu \nu}\left(\frac{P^{+}}{M}\right)^{2}\left(X_{2}+2 x X_{4}+x^{2} X_{3}\right)+\left(\frac{P^{+}}{M}\right)^{2} \frac{p_{T}^{\mu} p_{T}^{\nu}}{M^{2}} X_{6}+\frac{P^{+}}{M} \frac{p_{T}^{\mu} n_{-}^{\nu}+p_{T}^{\nu} n_{-}^{\mu}}{M}\left[X_{4}+x X_{3}+\left(\frac{\sigma}{2}-x\right) X_{6}\right] \\
& -\frac{P^{+}}{M} \frac{p_{T}^{[\mu} n_{-}^{\nu]}}{M} i X_{5}+n_{-}^{\mu} n_{-}^{\nu}\left[2 X_{1}+X_{2}+x X_{4}+x\left(X_{4}+x X_{3}\right)+2\left(\frac{\sigma}{2}-x\right)\left(X_{4}+x X_{3}\right)+\left(\frac{\sigma}{2}-x\right)^{2} X_{6}\right]
\end{aligned}
$$

where we have used $\sigma=2(P \cdot p) / M^{2}$ and $x=p^{+} / P^{+}$.

The twist-2 correlation function comes from transverse components $\mathcal{W}^{+i ;+j}$,

$$
\mathcal{W}^{+i ;+j}=-g_{T}^{i j}\left(\frac{P^{+}}{M}\right)^{2}\left(X_{2}+2 x X_{4}+x^{2} X_{3}\right)+\left(\frac{P^{+}}{M}\right)^{2} \frac{p_{T}^{i} p_{T}^{j}}{M^{2}} X_{6}
$$

Integrating over $p^{-}$for $\mathcal{W}^{+i ;+j}$, we have the following parametrization

$$
\int d p^{-} \mathcal{W}^{+i ;+j}(P, p)=\frac{1}{2} P^{+}\left[-g_{T}^{i j} x f^{\gamma}\left(x, \mathbf{p}_{T}^{2}\right)+\left(\frac{p_{T}^{i} p_{T}^{j}}{M^{2}}+\frac{\mathbf{p}_{T}^{2}}{2 M^{2}} g_{T}^{i j}\right) x h_{T}^{\gamma}\left(x, \mathbf{p}_{T}^{2}\right)\right],
$$

where $f^{\gamma}\left(x, \mathbf{p}_{T}^{2}\right)$ and $h_{T}^{\gamma}\left(x, \mathbf{p}_{T}^{2}\right)$ are distributions defined as

$$
\begin{aligned}
& x f^{\gamma}\left(x, \mathbf{p}_{T}^{2}\right)=\frac{2 P^{+}}{M^{2}} \int d p^{-}\left(X_{2}+2 x X_{4}+x^{2} X_{3}+\frac{\mathbf{p}_{T}^{2}}{2 M^{2}} X_{6}\right), \\
& x h_{T}^{\gamma}\left(x, \mathbf{p}_{T}^{2}\right)=\frac{2 P^{+}}{M^{2}} \int d p^{-} X_{6} .
\end{aligned}
$$

The twist- 3 contribution of $\mathcal{W}^{+\mu ;+\nu}$ comes from following components

$$
\begin{aligned}
\mathcal{W}^{+i ;+-} & =\frac{P^{+}}{M} \frac{p_{T}^{i}}{M}\left[X_{4}+x X_{3}+\left(\frac{\sigma}{2}-x\right) X_{6}-i X_{5}\right], \\
\mathcal{W}^{i j ; k+} & =\frac{-g_{T}^{k i} p_{T}^{j]}}{M} \frac{P^{+}}{M}\left(X_{4}+x X_{3}-i X_{5}\right) .
\end{aligned}
$$

The twist-4 contribution reads

$$
\mathcal{W}^{+-;+-}=2 X_{1}+X_{2}+x X_{4}+x\left(X_{4}+x X_{3}\right)+2\left(\frac{\sigma}{2}-x\right)\left(X_{4}+x X_{3}\right)+\left(\frac{\sigma}{2}-x\right)^{2} X_{6}
$$

More details and discussions can be found in Ref. [91] and references therein. 
Now we take the classical field approximation. We transform Eq. (C3) to momentum space and obtain

$$
\begin{aligned}
\mathcal{W}^{+\mu ;+\nu}(P, p) & =\frac{1}{(2 \pi)^{4}} \int \frac{d^{4} p^{\prime}}{(2 \pi)^{4}}\left\langle P\left|F^{+\mu}\left(p^{\prime}\right) F^{+\nu}(p)\right| P\right\rangle \\
& =\frac{1}{(2 \pi)^{4}} \int \frac{d^{4} p^{\prime}}{(2 \pi)^{4}}\left\langle P\left|\left[p^{\prime+} A^{\dagger \mu}\left(p^{\prime}\right)-p^{\prime \mu} A^{\dagger+}\left(p^{\prime}\right)\right]\left[p^{+} A^{\nu}(p)-p^{\nu} A^{+}(p)\right]\right| P\right\rangle \\
& =\frac{1}{(2 \pi)^{4}} \int \frac{d^{4} p^{\prime}}{(2 \pi)^{4}} p^{\prime+} p^{+}\left\langle P\left|A^{\dagger \mu}\left(p^{\prime}\right) A^{\nu}(p)\right| P\right\rangle,
\end{aligned}
$$

where we have used the light-cone gauge $A^{+}=0$. In the light-cone gauge the classical field has the form

$$
A^{\mu}(p)=2 \pi Z e \delta(p \cdot u) \frac{F\left(-p^{2}\right)}{-p^{2}}\left[u^{\mu}-\frac{p^{\mu}}{p^{+}} u^{+}\right]
$$

as the solution to the Maxwell equation. Here $u^{\mu}=\gamma(1,0,0, v)$ is the four-velocity of the nucleus satisfying $P^{\mu}=M u^{\mu}$. We use the same ansatz as (12) for the matrix element in Eq. (C9)

$$
\left\langle P\left|A^{\dagger \mu}\left(p^{\prime}\right) A^{\nu}(p)\right| P\right\rangle \approx 2 M A^{* \mu}\left(p^{\prime}\right) A^{\nu}(p)(2 \pi)^{3} \delta\left(p \cdot \bar{u}-p^{\prime} \cdot \bar{u}\right) \delta^{(2)}\left(\mathbf{p}_{T}-\mathbf{p}_{T}^{\prime}\right)
$$

where $\bar{u}^{\mu} \equiv \gamma(v, 0,0,1)$ satisfying $u \cdot \bar{u}=0$. Using the above formula in (C9) we obtain the transverse component

$$
\begin{aligned}
\mathcal{W}^{+i++j}(P, p)= & \frac{1}{2 \pi} \int \frac{d^{4} p^{\prime}}{(2 \pi)^{4}} p^{\prime+} p^{+} 2 M A^{* i}\left(p^{\prime}\right) A^{j}(p) \delta\left(p \cdot \bar{u}-p^{\prime} \cdot \bar{u}\right) \delta^{(2)}\left(\mathbf{p}_{T}-\mathbf{p}_{T}^{\prime}\right) \\
= & \frac{1}{2 \pi}(2 \pi Z e)^{2} 2 M \int \frac{d^{4} p^{\prime}}{(2 \pi)^{4}} p^{\prime+} p^{+} \delta\left(p^{\prime} \cdot u\right) \delta(p \cdot u) \frac{F^{*}\left(-p^{\prime 2}\right)}{-p^{\prime 2}} \frac{F\left(-p^{2}\right)}{-p^{2}}\left[u^{i}-\frac{p^{\prime}}{p^{\prime+}} u^{+}\right]\left[u^{j}-\frac{p^{j}}{p^{+}} u^{+}\right] \\
& \times \delta\left(p \cdot \bar{u}-p^{\prime} \cdot \bar{u}\right) \delta^{(2)}\left(\mathbf{p}_{T}-\mathbf{p}_{T}^{\prime}\right) \\
= & \frac{1}{4 \pi^{3}} M Z^{2} e^{2}\left(u^{+}\right)^{2} \delta(p \cdot u)\left|\frac{F\left(-p^{2}\right)}{-p^{2}}\right|^{2} p_{T}^{i} p_{T}^{j},
\end{aligned}
$$

where we have used $d^{4} p^{\prime}=d\left(p^{\prime} \cdot u\right) d\left(-p^{\prime} \cdot \bar{u}\right) d^{2} \mathbf{p}_{T}^{\prime}$ in carrying out the integral over $p^{\prime}$ and the constraints by the delta functions give $p^{\prime \mu}=p^{\mu}$.

Then we take an integral over $p^{-}$for $\mathcal{W}^{+i ;+j}(P, p)$ and obtain

$$
\int d p^{-} \mathcal{W}^{+i ;+j}(P, p)=\frac{1}{4 \pi^{3}} M Z^{2} e^{2} u^{+}\left|\frac{F\left(-p^{2}\right)}{-p^{2}}\right|^{2} p_{T}^{i} p_{T}^{j}
$$

where $p$ denotes the momentum that satisfies $p \cdot u=0$, in Sec. III we denote it as $\bar{p}$ as defined in Eq. (14), but here we suppressed the bar to simplify the notation. Comparing with Eq. (C5) we can extract

$$
\begin{aligned}
& x h_{T}^{\gamma}\left(x, \mathbf{p}_{T}^{2}\right)=\frac{2 Z^{2} \alpha}{\pi^{2}} M^{2}\left|\frac{F\left(-p^{2}\right)}{-p^{2}}\right|^{2} \\
& x f^{\gamma}\left(x, \mathbf{p}_{T}^{2}\right)=\frac{\mathbf{p}_{T}^{2}}{2 M^{2}} x h_{T}^{\gamma}\left(x, \mathbf{p}_{T}^{2}\right)=\frac{Z^{2} \alpha}{\pi^{2}} \mathbf{p}_{T}^{2}\left|\frac{F\left(-p^{2}\right)}{-p^{2}}\right|^{2}
\end{aligned}
$$

\section{APPENDIX D: INDEPENDENT VARIABLES FOR NUMERICAL INTEGRATION OF CROSS SECTIONS}

If the wave packets are very narrow in momentum we can make an approximation in Eq. $(20): G_{T}\left[\left(\mathbf{p}_{i T}^{\prime}-\mathbf{p}_{i T}\right)^{2}\right] \approx 1$ for $i=1,2$. In this case we can carry out the integrals over $\mathbf{b}_{1 T}, \mathbf{b}_{2 T}$, and $\mathbf{p}_{2 T}^{\prime}$ to obtain 


$$
\begin{aligned}
\sigma= & \frac{Z^{4} e^{4}}{2 \gamma^{4} v^{3}} \int d^{2} \mathbf{b}_{T} \int \frac{d \omega_{1} d^{2} \mathbf{p}_{1 T}}{(2 \pi)^{3}} \frac{d \omega_{2} d^{2} \mathbf{p}_{2 T}}{(2 \pi)^{3}} \frac{d^{2} \mathbf{p}_{1 T}^{\prime}}{(2 \pi)^{2}} e^{-i \mathbf{b}_{T} \cdot\left(\mathbf{p}_{1 T}^{\prime}-\mathbf{p}_{1 T}\right)} \frac{F^{*}\left(-p_{1}^{\prime 2}\right)}{-p_{1}^{\prime 2}} \frac{F\left(-p_{1}^{2}\right)}{-p_{1}^{2}} \frac{F^{*}\left(-p_{2}^{\prime 2}\right)}{-p_{2}^{\prime 2}} \frac{F\left(-p_{2}^{2}\right)}{-p_{2}^{2}} \\
& \times \int \frac{d^{3} k_{1}}{(2 \pi)^{3} 2 E_{k 1}} \frac{d^{3} k_{2}}{(2 \pi)^{3} 2 E_{k 2}} \sum_{\text {spin of } l, \bar{l}}\left[u_{1 \mu} u_{2 \nu} L^{\mu \nu}\left(p_{1}, p_{2} ; k_{1}, k_{2}\right)\right]\left[u_{1 \sigma} u_{2 \rho} L^{\sigma *}\left(p_{1}^{\prime}, p_{2}^{\prime} ; k_{1}, k_{2}\right)\right](2 \pi)^{4} \delta^{(4)}\left(p_{1}+p_{2}-k_{1}-k_{2}\right),
\end{aligned}
$$

where $\mathbf{p}_{2 T}^{\prime}=\mathbf{p}_{1 T}+\mathbf{p}_{2 T}-\mathbf{p}_{1 T}^{\prime}, \omega_{1}^{\prime}=\omega_{1}$, and $\omega_{2}^{\prime}=\omega_{2}$. Note that the photon momenta $p_{1}, p_{2}, p_{1}^{\prime}, p_{2}^{\prime}$ are momenta that satisfy $p_{i} \cdot u=0$ and $p_{i}^{\prime} \cdot u=0$ for $i=1,2$, in Sec. III we denote them as $\bar{p}_{1}, \bar{p}_{2}, \bar{p}_{1}^{\prime}, \bar{p}_{2}^{\prime}$ as defined in Eq. (14) but here we suppressed the bars to simplify the notation. Then we can complete the integrals over $\omega_{1}, \omega_{2}$ and $\mathbf{p}_{2 T}$ to remove the delta functions, fixing $\omega_{1}$ and $\omega_{2}$ as

$$
\begin{aligned}
& \omega_{1}=\frac{1}{2}\left[k_{1}^{0}+k_{2}^{0}+\left(k_{1}^{z}+k_{2}^{z}\right) v\right], \\
& \omega_{2}=\frac{1}{2}\left[k_{1}^{0}+k_{2}^{0}-\left(k_{1}^{z}+k_{2}^{z}\right) v\right],
\end{aligned}
$$

and fixing $\mathbf{p}_{2 T}$ and $\mathbf{p}_{2 T}^{\prime}$ as $\mathbf{p}_{2 T}=\mathbf{k}_{1 T}+\mathbf{k}_{2 T}-\mathbf{p}_{1 T}$ and $\mathbf{p}_{2 T}^{\prime}=\mathbf{k}_{1 T}+\mathbf{k}_{2 T}-\mathbf{p}_{1 T}^{\prime}$. This gives the cross section of the form

$$
\begin{aligned}
\sigma= & \frac{Z^{4} \alpha^{2}}{64 \pi^{4} \gamma^{4} v^{2}} \int d y_{1} d^{2} \mathbf{k}_{1 T} d y_{2} d^{2} \mathbf{k}_{2 T} \int d^{2} \mathbf{b}_{T} \int \frac{d^{2} \mathbf{p}_{1 T}}{(2 \pi)^{2}} \frac{d^{2} \mathbf{p}_{1 T}^{\prime}}{(2 \pi)^{2}} e^{-i \mathbf{b}_{T} \cdot\left(\mathbf{p}_{1 T}^{\prime}-\mathbf{p}_{1 T}\right)} \\
& \times \frac{F^{*}\left(-p_{1}^{\prime 2}\right)}{-p_{1}^{\prime 2}} \frac{F\left(-p_{1}^{2}\right)}{-p_{1}^{2}} \frac{F^{*}\left(-p_{2}^{\prime 2}\right)}{-p_{2}^{\prime 2}} \frac{F\left(-p_{2}^{2}\right)}{-p_{2}^{2}} \sum_{\text {spin of } l, \bar{l}}\left[u_{1 \mu} u_{2 \nu} L^{\mu \nu}\left(p_{1}, p_{2} ; k_{1}, k_{2}\right)\right]\left[u_{1 \sigma} u_{2 \rho} L^{\sigma \rho *}\left(p_{1}^{\prime}, p_{2}^{\prime} ; k_{1}, k_{2}\right)\right],
\end{aligned}
$$

where we have used the rapidity and transverse momentum as independent variables for lepton momenta so that the lepton momenta $k_{1}$ and $k_{2}$ can be expressed as

$$
k^{\mu}=\frac{m_{T}}{\sqrt{2}} e^{y} n_{+}^{\mu}+\frac{m_{T}}{\sqrt{2}} e^{-y} n_{-}^{\mu}+k_{T}^{\mu} .
$$

Here the rapidity and transverse mass are defined as

$$
\begin{aligned}
y & =\frac{1}{2} \ln \frac{E+k_{z}}{E-k_{z}}=\frac{1}{2} \ln \frac{k^{+}}{k^{-}}, \\
m_{T}^{2} & =\mathbf{k}_{T}^{2}+m^{2}=E^{2}-k_{z}^{2}=2 k^{+} k^{-} .
\end{aligned}
$$

Then we have $d^{3} k / E_{k}=d y d^{2} \mathbf{k}_{T}$.

Now we choose independent integration variables of the cross section. The impact parameter is written as

$$
\mathbf{b}_{T}=\left(b_{T} \cos \phi_{b}, b_{T} \sin \phi_{b}\right)
$$

The transverse momentum shift is defined as

$$
\boldsymbol{\Delta}_{T} \equiv \mathbf{p}_{1 T}^{\prime}-\mathbf{p}_{1 T}=\left(\Delta_{T} \cos \phi_{\Delta}, \Delta_{T} \sin \phi_{\Delta}\right)
$$

so the integral over $\mathbf{p}_{1 T}^{\prime}$ can be replaced by $\boldsymbol{\Delta}_{T}$. Since the invariant mass and transverse momentum spectra of lepton pairs were measured in experiments, we should define the four-momentum of the lepton pair

$$
\begin{aligned}
P_{e e}^{\mu} & =k_{1}^{\mu}+k_{2}^{\mu}=\left(P_{e e}^{0}, \mathbf{P}_{e e}^{T}, P_{e e}^{z}\right) \\
& =\left(\sqrt{M_{e e}^{2}+\left(P_{e e}^{T}\right)^{2}} \cosh Y_{e e}, P_{e e}^{T} \cos \phi_{e e}, P_{e e}^{T} \sin \phi_{e e}, \sqrt{M_{e e}^{2}+\left(P_{e e}^{T}\right)^{2}} \sinh Y_{e e}\right)
\end{aligned}
$$

where $Y_{e e}$ is the rapidity of the lepton pair and $M_{e e}$ is the invariant mass of the lepton pair given by 


$$
M_{e e}^{2}=P_{e e}^{2}=2 m_{e}^{2}+2 k_{1} \cdot k_{2}=2 P_{e e} \cdot k_{2} .
$$

Instead of $k_{1}^{\mu}$ and $k_{2}^{\mu}$, we choose $P_{e e}^{\mu}$ and two variables of $k_{2}^{\mu}$ as independent variables since $k_{2}^{\mu}$ satisfies the constraint (D9). Two variables of $k_{2}^{\mu}$ are chosen to be the pseudorapidity $\eta_{k 2}$ and the azimuthal angle $\phi_{k 2}$ of the transverse momentum, so $k_{2}^{\mu}$ can be expressed by

$$
k_{2}^{\mu}=\left(\sqrt{m_{e}^{2}+k_{2 T}^{2} \cosh ^{2} \eta_{k 2}}, k_{2 T} \cos \phi_{k 2}, k_{2 T} \sin \phi_{k 2}, k_{2 T} \sinh \eta_{k 2}\right) .
$$

Here $k_{2 T}$ is obtained by solving Eq. (D9),

$$
k_{2 T}=\frac{f_{1} M_{e e}^{2}+\sqrt{f_{1}^{2} M_{e e}^{4}-f_{2} f_{3}}}{f_{2}},
$$

where

$$
\begin{aligned}
& f_{1}=2\left(P_{e e}^{T} \cos \phi_{e e} \cos \phi_{k 2}+q_{T} \sin \phi_{e e} \sin \phi_{k 2}+\sqrt{M_{e e}^{2}+\left(P_{e e}^{T}\right)^{2}} \sinh Y_{e e} \sinh \eta_{k 2}\right), \\
& f_{2}=4\left[M_{e e}^{2}+\left(P_{e e}^{T}\right)^{2}\right] \cosh ^{2} Y_{e e} \cosh ^{2} \eta_{k 2}-f_{1}^{2}, \\
& f_{3}=-M_{e e}^{4}+4\left[M_{e e}^{2}+\left(P_{e e}^{T}\right)^{2}\right] m_{e}^{2} \cosh ^{2} Y_{e e} .
\end{aligned}
$$

One can verify

$$
d y_{1} d^{2} k_{1 T} d y_{2} d^{2} k_{2 T}=\mathcal{J} d Y_{e e} d P_{e e}^{T} d \phi_{e e} d M_{e e} d \eta_{k 2} d \phi_{k 2},
$$

where $\mathcal{J}$ is a function of $P_{e e}^{\mu}$ and $k_{2}^{\mu}$. From $P_{e e}^{\mu}$ and $k_{2}^{\mu}$, we obtain $k_{1}^{\mu}=P_{e e}^{\mu}-k_{2}^{\mu}$. The photon momenta are given by

$$
\begin{aligned}
& p_{1}^{\mu}=\left(\frac{P_{e e}^{0}+v P_{e e}^{z}}{2}, p_{1 T} \cos \phi_{p 1}, p_{1 T} \sin \phi_{p 1}, \frac{P_{e e}^{0}+v P_{e e}^{z}}{2 v}\right), \\
& p_{2}^{\mu}=\left(\frac{P_{e e}^{0}-v P_{e e}^{z}}{2}, P_{e e}^{x}-p_{1 T} \cos \phi_{p 1}, P_{e e}^{y}-p_{1 T} \sin \phi_{p 1},-\frac{P_{e e}^{0}-v P_{e e}^{z}}{2 v}\right), \\
& p_{1}^{\prime \mu}=\left(\frac{P_{e e}^{0}+v P_{e e}^{z}}{2}, p_{1 T} \cos \phi_{p 1}+\Delta_{T} \cos \phi_{\Delta}, p_{1 T} \sin \phi_{p 1}+\Delta_{T} \sin \phi_{\Delta}, \frac{P_{e e}^{0}+v P_{e e}^{z}}{2 v}\right), \\
& p_{2}^{\prime \mu}=\left(\frac{P_{e e}^{0}-v P_{e e}^{z}}{2}, P_{e e}^{x}-p_{1 T} \cos \phi_{p 1}-\Delta_{T} \cos \phi_{\Delta}, P_{e e}^{y}-p_{1 T} \sin \phi_{p 1}-\Delta_{T} \sin \phi_{\Delta},-\frac{P_{e e}^{0}-v P_{e e}^{z}}{2 v}\right),
\end{aligned}
$$

where we can choose $p_{1 T}$ and $\phi_{p 1}$ as independent variables.

In summary, we can choose the following integration variables:

$$
\left(Y_{e e}, P_{e e}^{T}, \phi_{e e}, M_{e e}\right),\left(\eta_{k 2}, \phi_{k 2}\right),\left(p_{1 T}, \phi_{p 1}\right),\left(\Delta_{T}, \phi_{\Delta}\right),\left(b_{T}, \phi_{b}\right),
$$

where we used the parentheses to enclose variables from the same source. In terms of these independent variables, the cross section (D3) can be rewritten in the form of Eq. (48). 
[1] D. E. Kharzeev, L. D. McLerran, and H. J. Warringa, Nucl. Phys. A803, 227 (2008).

[2] V. Skokov, A. Y. Illarionov, and V. Toneev, Int. J. Mod. Phys. A 24, 5925 (2009).

[3] A. Bzdak and V. Skokov, Phys. Lett. B 710, 171 (2012).

[4] V. Voronyuk, V. D. Toneev, W. Cassing, E. L. Bratkovskaya, V. P. Konchakovski, and S. A. Voloshin, Phys. Rev. C 83, 054911 (2011).

[5] W.-T. Deng and X.-G. Huang, Phys. Rev. C 85, 044907 (2012).

[6] V. Roy and S. Pu, Phys. Rev. C 92, 064902 (2015).

[7] H. Li, X.-L. Sheng, and Q. Wang, Phys. Rev. C 94, 044903 (2016).

[8] G. Inghirami, L. Del Zanna, A. Beraudo, M. H. Moghaddam, F. Becattini, and M. Bleicher, Eur. Phys. J. C 76, 659 (2016).

[9] I. Siddique, X.-L. Sheng, and Q. Wang, arXiv:2106.00478.

[10] S. Pu, V. Roy, L. Rezzolla, and D. H. Rischke, Phys. Rev. D 93, 074022 (2016).

[11] V. Roy, S. Pu, L. Rezzolla, and D. Rischke, Phys. Lett. B 750, 45 (2015).

[12] S. Pu and D.-L. Yang, EPJ Web Conf. 137, 13021 (2017).

[13] M. Shokri and N. Sadooghi, J. High Energy Phys. 11 (2018) 181.

[14] I. Siddique, R.-j. Wang, S. Pu, and Q. Wang, Phys. Rev. D 99, 114029 (2019).

[15] R.-J. Wang, P. Copinger, and S. Pu, Nucl. Phys. A1005, 121869 (2021).

[16] A. Vilenkin, Phys. Rev. D 22, 3080 (1980).

[17] K. Fukushima, D. E. Kharzeev, and H. J. Warringa, Phys. Rev. D 78, 074033 (2008).

[18] X.-G. Huang and J. Liao, Phys. Rev. Lett. 110, 232302 (2013).

[19] S. Pu, S.-Y. Wu, and D.-L. Yang, Phys. Rev. D 89, 085024 (2014).

[20] Y. Jiang, X.-G. Huang, and J. Liao, Phys. Rev. D 91, 045001 (2015).

[21] S. Pu, S.-Y. Wu, and D.-L. Yang, Phys. Rev. D 91, 025011 (2015).

[22] J.-W. Chen, T. Ishii, S. Pu, and N. Yamamoto, Phys. Rev. D 93, 125023 (2016).

[23] Y. Hidaka, S. Pu, and D.-L. Yang, Phys. Rev. D 97, 016004 (2018).

[24] S. Ebihara, K. Fukushima, and S. Pu, Phys. Rev. D 96, 016016 (2017).

[25] J.-W. Chen, J.-H. Gao, J. Liu, S. Pu, and Q. Wang, Phys. Rev. D 88, 074003 (2013).

[26] M. A. Stephanov and Y. Yin, Phys. Rev. Lett. 109, 162001 (2012).

[27] J.-W. Chen, J.-y. Pang, S. Pu, and Q. Wang, Phys. Rev. D 89, 094003 (2014).

[28] J.-Y. Chen, D. T. Son, M. A. Stephanov, H.-U. Yee, and Y. Yin, Phys. Rev. Lett. 113, 182302 (2014).

[29] D. T. Son and N. Yamamoto, Phys. Rev. Lett. 109, 181602 (2012).

[30] D. T. Son and N. Yamamoto, Phys. Rev. D 87, 085016 (2013).

[31] J.-H. Gao, Z.-T. Liang, S. Pu, Q. Wang, and X.-N. Wang, Phys. Rev. Lett. 109, 232301 (2012).
[32] J.-W. Chen, S. Pu, Q. Wang, and X.-N. Wang, Phys. Rev. Lett. 110, 262301 (2013).

[33] J.-h. Gao and Q. Wang, Phys. Lett. B 749, 542 (2015).

[34] Y. Hidaka, S. Pu, and D.-L. Yang, Phys. Rev. D 95, 091901 (2017).

[35] J.-H. Gao, S. Pu, and Q. Wang, Phys. Rev. D 96, 016002 (2017).

[36] Y. Hidaka, S. Pu, and D.-L. Yang, Nucl. Phys. A982, 547 (2019).

[37] J.-H. Gao, Z.-T. Liang, Q. Wang, and X.-N. Wang, Phys. Rev. D 98, 036019 (2018).

[38] A. Huang, S. Shi, Y. Jiang, J. Liao, and P. Zhuang, Phys. Rev. D 98, 036010 (2018).

[39] N. Mueller and R. Venugopalan, Phys. Rev. D 97, 051901 (2018).

[40] J.-H. Gao and Z.-T. Liang, Phys. Rev. D 100, 056021 (2019).

[41] N. Weickgenannt, X.-L. Sheng, E. Speranza, Q. Wang, and D. H. Rischke, Phys. Rev. D 100, 056018 (2019).

[42] N. Weickgenannt, E. Speranza, X.-1. Sheng, Q. Wang, and D. H. Rischke, Phys. Rev. Lett. 127, 052301 (2021).

[43] N. Weickgenannt, X.-L. Sheng, E. Speranza, Q. Wang, and D. H. Rischke, Wigner function and kinetic theory for massive spin-1/2 particles, in 28th International Conference on Ultrarelativistic Nucleus-Nucleus Collisions (Quark Matter 2019) Wuhan, China, 2019, 2020, arXiv:2001.11862.

[44] K. Hattori, Y. Hidaka, and D.-L. Yang, Phys. Rev. D 100, 096011 (2019).

[45] D.-L. Yang, K. Hattori, and Y. Hidaka, J. High Energy Phys. 07 (2020) 070.

[46] Y.-C. Liu, K. Mameda, and X.-G. Huang, Chin. Phys. C 44, 094101 (2020).

[47] N. Weickgenannt, E. Speranza, X.-L. Sheng, Q. Wang, and D. H. Rischke, Phys. Rev. D 104, 016022 (2021).

[48] X.-L. Sheng, N. Weickgenannt, E. Speranza, D. H. Rischke, and Q. Wang, Phys. Rev. D 104, 016029 (2021).

[49] J. Liao, Pramana 84, 901 (2015).

[50] D. E. Kharzeev, Annu. Rev. Nucl. Part. Sci. 65, 193 (2015).

[51] X.-G. Huang, Rep. Prog. Phys. 79, 076302 (2016).

[52] J.-H. Gao, G.-L. Ma, S. Pu, and Q. Wang, Nucl. Sci. Technol. 31, 90 (2020).

[53] Y.-C. Liu and X.-G. Huang, Nucl. Sci. Technol. 31, 56 (2020).

[54] J. S. Schwinger, Phys. Rev. 82, 664 (1951).

[55] K. Fukushima, D. E. Kharzeev, and H. J. Warringa, Phys. Rev. Lett. 104, 212001 (2010).

[56] H. J. Warringa, Phys. Rev. D 86, 085029 (2012).

[57] P. Copinger, K. Fukushima, and S. Pu, Phys. Rev. Lett. 121, 261602 (2018).

[58] P. Copinger and S. Pu, Int. J. Mod. Phys. A 35, 203005 (2020).

[59] K. Hattori and K. Itakura, Ann. Phys. (Amsterdam) 330, 23 (2013).

[60] K. Hattori and K. Itakura, Ann. Phys. (Amsterdam) 334, 58 (2013).

[61] K. Hattori, H. Taya, and S. Yoshida, J. High Energy Phys. 01 (2021) 093.

[62] C. F. von Weizsacker, Z. Phys. 88, 612 (1934).

[63] E. J. Williams, Phys. Rev. 45, 729 (1934). 
[64] J.D. Jackson, Classical Electrodynamics (Wiley, New York, 1998).

[65] G. Breit and J. A. Wheeler, Phys. Rev. 46, 1087 (1934).

[66] J. Adams et al. (STAR Collaboration), Phys. Rev. C 70, 031902 (2004).

[67] J. Adam et al. (STAR Collaboration), Phys. Rev. Lett. 127, 052302 (2021).

[68] M. Aaboud et al. (ATLAS Collaboration), Nat. Phys. 13, 852 (2017).

[69] J. Adam et al. (STAR Collaboration), Phys. Rev. Lett. 121, 132301 (2018).

[70] M. Aaboud et al. (ATLAS Collaboration), Phys. Rev. Lett. 121, 212301 (2018).

[71] M. Vidovic, M. Greiner, C. Best, and G. Soff, Phys. Rev. C 47, 2308 (1993).

[72] A. J. Baltz, Y. Gorbunov, S. R. Klein, and J. Nystrand, Phys. Rev. C 80, 044902 (2009).

[73] S. R. Klein, J. Nystrand, J. Seger, Y. Gorbunov, and J. Butterworth, Comput. Phys. Commun. 212, 258 (2017).

[74] W. Zha, L. Ruan, Z. Tang, Z. Xu, and S. Yang, Phys. Lett. B 781, 182 (2018).

[75] K. Hencken, D. Trautmann, and G. Baur, Phys. Rev. A 51, 1874 (1995)

[76] K. Hencken, G. Baur, and D. Trautmann, Phys. Rev. C 69, 054902 (2004).

[77] W. Zha, J. D. Brandenburg, Z. Tang, and Z. Xu, Phys. Lett. B 800, 135089 (2020).

[78] J. D. Brandenburg et al., arXiv:2006.07365.

[79] W. Zha and Z. Tang, arXiv:2103.04605.

[80] C. Li, J. Zhou, and Y.-J. Zhou, Phys. Lett. B 795, 576 (2019).

[81] C. Li, J. Zhou, and Y.-J. Zhou, Phys. Rev. D 101, 034015 (2020).

[82] A. Metz and J. Zhou, Phys. Rev. D 84, 051503 (2011).

[83] E. Akcakaya, A. Schäfer, and J. Zhou, Phys. Rev. D 87, 054010 (2013).

[84] C. Pisano, D. Boer, S. J. Brodsky, M. G. A. Buffing, and P. J. Mulders, J. High Energy Phys. 10 (2013) 024.

[85] S. Klein, A. H. Mueller, B.-W. Xiao, and F. Yuan, Phys. Rev. D 102, 094013 (2020).

[86] B.-W. Xiao, F. Yuan, and J. Zhou, Phys. Rev. Lett. 125, 232301 (2020).
[87] S. Klein, A. H. Mueller, B.-W. Xiao, and F. Yuan, Phys. Rev. Lett. 122, 132301 (2019).

[88] F. Krauss, M. Greiner, and G. Soff, Prog. Part. Nucl. Phys. 39, 503 (1997).

[89] Y. Shi, L. Wang, S.-Y. Wei, B.-W. Xiao, and L. Zheng, Phys. Rev. D 103, 054017 (2021).

[90] F. Ringer, B.-W. Xiao, and F. Yuan, Phys. Lett. B 808, 135634 (2020).

[91] P. J. Mulders and J. Rodrigues, Phys. Rev. D 63, 094021 (2001).

[92] S. J. Brodsky, H.-C. Pauli, and S. S. Pinsky, Phys. Rep. 301, 299 (1998).

[93] X. Ji, Y.-S. Liu, Y. Liu, J.-H. Zhang, and Y. Zhao, arXiv: 2004.03543.

[94] J. C. Collins and D. E. Soper, Nucl. Phys. B194, 445 (1982).

[95] J. C. Collins, D. E. Soper, and G. F. Sterman, Nucl. Phys. B250, 199 (1985).

[96] R. D. Tangerman and P. J. Mulders, Phys. Rev. D 51, 3357 (1995).

[97] A. Bacchetta, D. Boer, M. Diehl, and P. J. Mulders, J. High Energy Phys. 08 (2008) 023.

[98] X.-D. Ji, J.-P. Ma, and F. Yuan, Phys. Rev. D 71, 034005 (2005).

[99] H.-Z. Wu, J.-J. Zhang, L.-G. Pang, and Q. Wang, Comput. Phys. Commun. 248, 106962 (2020).

[100] J.-J. Zhang and H.-Z. Wu, Comput. Phys. Commun. 251, 107240 (2020).

[101] J.-J. Zhang, H.-Z. Wu, S. Pu, G.-Y. Qin, and Q. Wang, Phys. Rev. D 102, 074011 (2020).

[102] S. Klein and J. Nystrand, Phys. Rev. C 60, 014903 (1999).

[103] C. A. Bertulani and G. Baur, Phys. Rep. 163, 299 (1988).

[104] G. Baur, K. Hencken, and D. Trautmann, J. Phys. G 24, 1657 (1998).

[105] I. A. Pshenichnov, J. P. Bondorf, I. N. Mishustin, A. Ventura, and S. Masetti, Phys. Rev. C 64, 024903 (2001).

[106] A. J. Baltz, M. J. Rhoades-Brown, and J. Weneser, Phys. Rev. E 54, 4233 (1996).

[107] Z.-H. Sun, D.-X. Zheng, J. Zhou, and Y.-J. Zhou, Phys. Lett. B 808, 135679 (2020).

[108] M. E. Peskin and D. V. Schroeder, An Introduction to Quantum Field Theory (Addison-Wesley, Reading, USA, 1995). 